\title{
Corticolimbic Transcriptome Changes are State-Dependent and Region-Specific in a Rodent Model of Depression and of Antidepressant Reversal
}

\author{
Alexandre Surget', Yingjie Wang ${ }^{2}$, Samuel Leman', Yadira Ibarguen-Vargas', Nicole Edgar ${ }^{2,3}$, Guy Griebel $^{4}$, \\ Catherine Belzung' ${ }^{\prime}$ and Etienne Sibille ${ }^{*, 2,3}$ \\ 'U930 FRE CNRS 2448, INSERM and Université François Rabelais, Tours, France; '2Department of Psychiatry, University of Pittsburgh, Pittsburgh, \\ PA, USA; ${ }^{3}$ Center For Neuroscience, University of Pittsburgh, Pittsburgh, PA, USA; ${ }^{4}$ CNS Research Department, Sanofi-Aventis, Bagneux, France
}

\begin{abstract}
Gene microarrays may enable the elucidation of neurobiological changes underlying the pathophysiology and treatment of major depression. However, previous studies of antidepressant treatments were performed in healthy normal rather than 'depressed' animals. Since antidepressants are devoid of mood-changing effects in normal individuals, the clinically relevant rodent transcriptional changes could remain undetected. We investigated antidepressant-related transcriptome changes in a corticolimbic network of mood regulation in the context of the unpredictable chronic mild stress (UCMS), a naturalistic model of depression based on socio-environmental stressors. Mice subjected to a 7-week UCMS displayed a progressive coat state deterioration, reduced weight gain, and increased agonistic and emotion-related behaviors. Chronic administration of an effective (fluoxetine) or putative antidepressant (corticotropinreleasing factor-I (CRF $)$ antagonist, SSR I25543) reversed all physical and behavioral effects. Changes in gene expression differed among cingulate cortex (CC), amygdala (AMY) and dentate gyrus (DG) and were extensively reversed by both drugs in CC and AMY, and to a lesser extent in DG. Fluoxetine and SSRI 25543 also induced additional and very similar molecular profiles in UCMS-treated mice, but the effects of the same drug differed considerably between control and UCMS states. These studies established on a large-scale that the molecular impacts of antidepressants are region-specific and state-dependent, revealed common transcriptional changes downstream from different antidepressant treatments and supported CRF, targeting as an effective therapeutic strategy. Correlations between UCMS, drug treatments, and gene expression suggest distinct AMY neuronal and oligodendrocyte molecular phenotypes as candidate systems for mood regulation and therapeutic interventions.

Neuropsychopharmacology (2009) 34, I363-1380; doi:I0. I038/npp.2008.76; published online 4 June 2008
\end{abstract}

Keywords: stress; serotonin; CRF; transcriptome; amygdala; cingulate

\section{INTRODUCTION}

Although depression is a leading cause of disability worldwide and a serious health problem, mechanisms underlying its pathophysiology remain poorly characterized (Wong and Licinio, 2001; Nestler et al, 2002; Belmaker and Agam, 2008). Monoaminergic imbalances participate in the pathogenesis of depression, and response to antidepressant treatment is associated with increased monoaminergic neurotransmission (Duman et al, 1997; Manji et al, 2001; Nestler et al, 2002). However, the rapid antidepressantinduced neurochemical changes do not parallel the 2-4 weeks of drug exposure required for therapeutic effects,

\footnotetext{
*Correspondence: Dr E Sibille, Department of Psychiatry, University of Pittsburgh, 381 I O'Hara Street, BST W 1643, Pittsburgh, PA 152132593, USA, Tel: + 412624 0804, E-mail: sibilleel@upmc.edu

Received 19 February 2008; accepted 25 April 2008
}

indicating that downstream events must occur (Duman et al, 1997, 1999; Manji et al, 2001; Nestler et al, 2002). Indeed, the pathophysiology and treatment of depression induce biological events of greater complexity than changes in monoamine levels, including (1) structural and ultrastructural changes, altered synaptic, glial, or neuronal density, as reported in frontal/cingulate cortex (CC), amygdala (AMY), and hippocampus (Drevets et al, 1997; Sheline et al, 1998; Rajkowska et al, 1999; Bowley et al, 2002), (2) variations in neurotrophic factors and neurotransmitters systems (Duman et al, 1997; Castren, 2004), and (3) altered signal-transduction pathways (Manji et al, 2001; Shelton, 2007), which together may affect gene regulation through transcription factors or chromatin modifications (Tsankova et al, 2006).

Several gene microarray studies have attempted to characterize the molecular correlates of antidepressant treatment in rodents (Landgrebe et al, 2002; Rausch et al, 2002; Newton et al, 2003; Drigues et al, 2003; Palotas et al, 
2004; Altar et al, 2004; Wong et al, 2004; Ploski et al, 2006; Takahashi et al, 2006); however, differences in treatments, exposure time, and brain regions investigated have yielded no consensus. Moreover, Conti et al (2007) recently reported that changes in gene transcripts after three different antidepressant modalities in normal control rats were mostly brain region-specific. Importantly, these previous array studies were performed in healthy normal rather than 'depressed' animals, with the underlying assumption that antidepressant effects may be similar to those occurring in depressive-like states.

The unpredictable chronic mild stress (UCMS) is an informative model to study depression in animals (Willner, 2005), as it mimics in a naturalistic way the role of socio-environmental stressors in precipitating a depressive pathology and the time frame of therapeutic responses to antidepressants. Specifically, the random application of various environmental and social mild stressors for several weeks results in a syndrome in mice that is reminiscent of symptoms of depression, including increased fearfulness/ anxiety-like behavior, decreased consumption of palatable food, and physiological changes (Santarelli et al, 2003; Pothion et al, 2004; Mineur et al, 2006). Here, we investigated transcriptome changes in UCMS-treated mice, and after reversal by chronic exposure to a selective serotonin reuptake inhibitor (fluoxetine) or a corticotropin-releasing factor-1 $\left(\mathrm{CRF}_{1}\right)$ antagonist (SSR125543). $\mathrm{CRF}_{1}$ antagonists target a non-monoaminergic system that directly affects the stress pathway and display promising antidepressant profiles in preclinical tests and clinical trials (Zobel et al, 2000; Griebel et al, 2002; Kunzel et al, 2003; Ising et al, 2007; Surget et al, 2008). Currently, no single brain area has been identified as a primary region affected in depression; however, meta-analyses of altered brain function point toward a corticolimbic circuitry of mood regulation that is affected in depression (Mayberg, 1997; Seminowicz et al, 2004). This circuitry includes areas of the prefrontal cortex, the ACC, the hippocampus, anterior thalamic nuclei and the AMY. Thus, we focused on three rodent equivalent brain areas within this corticolimbic network: CC, AMY, and dentate gyrus (DG).

Here, we confirm that the physical and behavioral effects of UCMS are effectively reversed by chronic exposure to both drugs, and demonstrate that the molecular correlates of UCMS and antidepressant treatments are state-dependent and brain region-specific, thus confirming our hypothesis that antidepressant effects in control animals do not extrapolate to 'depressive states.' Our studies revealed different sets of putative therapeutic targets for each brain area, which may reflect functional differences between areas within a neural network of mood regulation.

\section{MATERIALS AND METHODS}

\section{Animals}

Male BALB/c mice ( 8 weeks old) (Centre d'élevage Janvier, Le Genest Saint Isle, France) were group-housed ( $n=4-5$ per cage) under standard conditions (12/12 h light-dark cycle, $22 \pm 1{ }^{\circ} \mathrm{C}$, food and water ad libitum) for 3 weeks prior to the experiments. To avoid possible bias due to acute effect of behavioral testing on gene expression, the experiment required two sets of mice: the first one $(n=18-$ 19 per group) supplied physical data and tissue samples for microarray and PCR analyses, while the second one ( $n=11-12$ per group) provided behavioral data. All animal care and treatment were in accordance with the European Community Council directive 86/609/EEC and with the Guide for Care and Use of Laboratory Animals established by the US National Institute of Health.

\section{Drugs}

The selective serotonin reuptake inhibitor fluoxetine (Eli Lilly, Indianapolis, IN, USA) and the $\mathrm{CRF}_{1}$ antagonist SSR125543 (Sanofi-Aventis, Bagneux, France) were prepared in saline $(\mathrm{NaCl} 0.9 \%)$ containing $5 \%$ dimethyl sulfoxide and $5 \%$ cremophor EL. Vehicle, fluoxetine $(20 \mathrm{mg} / \mathrm{kg} /$ day $)$ and SSR125543 (20 mg/kg/day) were administered intraperitoneally (i.p.), based on previous experiments (Griebel et al, 2002; Alonso et al, 2004; Kulkarni and Dhir, 2007). Concentrations were adjusted to administer $10 \mathrm{ml} / \mathrm{kg}$.

\section{UCMS}

Mice were subjected to various stressors according to a 'random' schedule for 7 weeks (Figure 1). UCMS-exposed mice were maintained under standard laboratory conditions but were isolated in small individual cages $(24 \mathrm{~cm} \times$ $11 \mathrm{~cm} \times 12 \mathrm{~cm}$ ), while non-stressed controls were grouphoused in standard laboratory cages $(42 \mathrm{~cm} \times 28 \mathrm{~cm} \times$ $18 \mathrm{~cm})$. Drug or vehicle treatments started on day 14 and stopped the day after the end of UCMS (day 50). The stressors were: altered bedding (sawdust change, removal, or damp; substitution of sawdust with $21^{\circ} \mathrm{C}$ water, rat, or cat feces); cage tilting $\left(45^{\circ}\right)$ or shaking $(2 \times 30 \mathrm{sec})$; cage exchange (mice positioned in the empty cage of another male); induced defensive posture (repeated slight grips on the back until the mouse showed a defensive posture) and altered length and time of light-dark cycle. Body weight and coat state were assessed weekly, as markers of the progression of the UCMS-evoked syndrome. The total score resulted from the sum of scores obtained from the head, neck, dorsal coat, ventral coat, tail, forepaws, and hindpaws $(0=$ well-groomed, $1=$ unkempt). This index has been pharmacologically validated (Griebel et al, 2002; Santarelli et al, 2003; Ducottet et al, 2003; Surget et al, 2008).

\section{Novelty-Suppressed Feeding Test}

The novelty-suppressed feeding (NSF) test was modified from Santarelli et al (2003) and performed after 45 days of UCMS. The testing apparatus consisted of a wooden box $(33 \mathrm{~cm} \times 33 \mathrm{~cm} \times 30 \mathrm{~cm})$ with an indirect red light. The floor was covered with $2 \mathrm{~cm}$ sawdust. At $12 \mathrm{~h}$ before the test, food was removed from the cages. At the time of testing, a single pellet of regular chow was placed on a white paper in the center of the box. An animal was placed in a corner of the box. The latency to manifestly chew the pellet was recorded for $3 \mathrm{~min}$. This test induces a conflict between the drive to eat and the fear of venturing into the open center. To control for potential antidepressant effects on appetite, we 

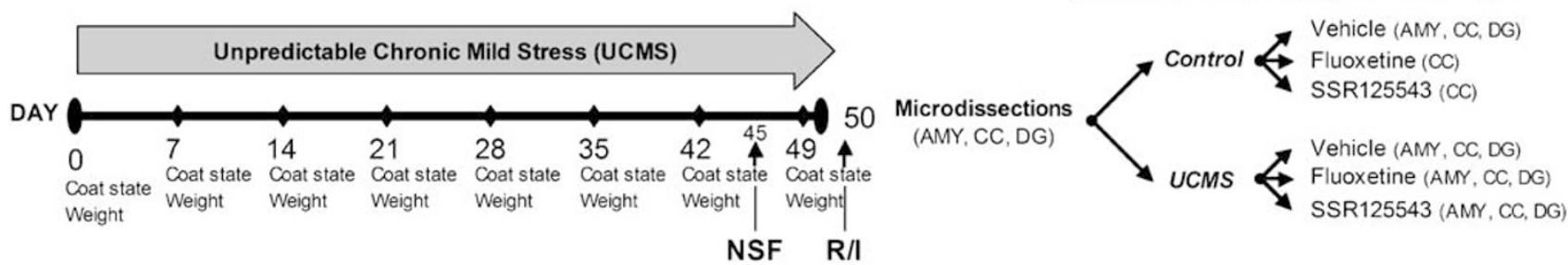

b

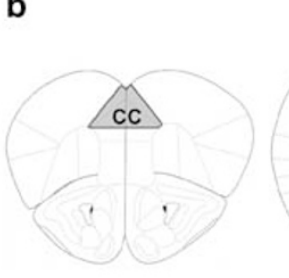

Bregma 2.4

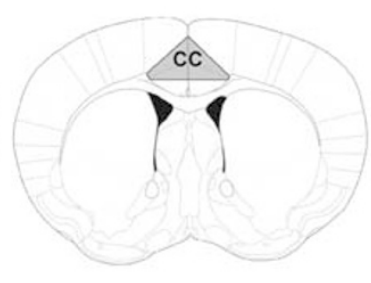

Bregma $\sim 0.4$

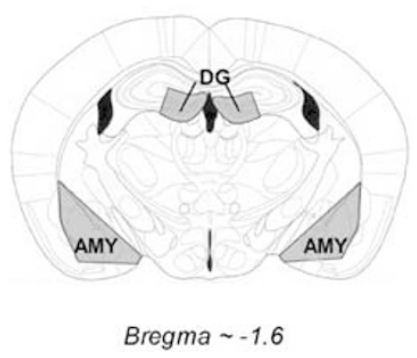

Bregma -1.6

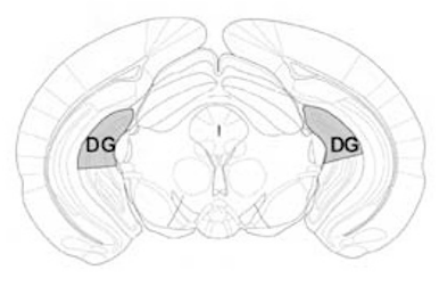

Bregma -3.6

Figure I Experimental design. (a) Six groups of mice ( $n=18-19$ mice per group) were used depending on the environment (control/UCMS) and the treatment (vehicle/fluoxetine/SSR I 25543). The UCMS regimen lasted 7 weeks. The coat state was evaluated and the body weight was measured weekly by two experimenters blind to the treatment. Drug or vehicle treatments started on day 14 and continued until the end of UCMS on day 50. Fluoxetine $(20 \mathrm{mg} / \mathrm{kg} / \mathrm{day})$, SSR I $25543(20 \mathrm{mg} / \mathrm{kg} /$ day), or vehicle were administered intraperitoneally once a day to UCMS-exposed or control mice. Toward the end of the UCMS regimen, six mice per group were chosen in such a way that the mice were representative of their group in terms of physical state and behavior. Amygdala (AMY), dentate gyrus (DG), and cingulate cortex (CC) were collected for microarray analysis from these six mice per group the day after the end of UCMS. Since behavioral testing would interfere with the 'control' state of control groups, acute behavioral testing was performed on independent groups ( $n=11-12$ mice per group) subjected to the same UCMS protocol. Tests included the novelty suppressed feeding (NSF) test at day 45 and the resident/intruder $(\mathrm{R} / \mathrm{l})$ test at day 50 . NSF and R/I tests were only performed once, to avoid interferences of short inter-test time periods. (b) Representative figures of the brain regions dissected (see Materials and methods). Figures are adapted from Paxinos and Franklin (200 I).

measured food consumption over 5 min after mice returned to their home cage.

\section{Resident/Intruder Test}

The resident/intruder (R/I) test was modified from previously described protocols (Guillot et al, 1994; Mineur et al, 2003) and was performed after 50 days of UCMS. Control mice were single-housed 2 days before testing. UCMS-treated mice were also placed in new cages 2 days prior to testing. All mice were tested against a 6-month-old intruder. The opponent was placed into the home cage of the test animal (resident) so that mice were in opposite corners. Latencies of the first attack and number of attacks were recorded for $10 \mathrm{~min}$. Attacking intruder mice were excluded.

\section{Behavioral Data Statistical Analysis}

The effects on physical and behavioral states of 7-week UCMS (environment), 5-week antidepressant treatments, and their interactions were evaluated by ANOVAs. Significant main effects or interaction were followed up with post hoc Tukey test (HSD for $n$ different), where appropriate.

\section{Brain Area Sampling}

Brain areas were collected at the time of maximum UCMS and antidepressant effects (7 weeks of UCMS and 5 weeks of treatment) and $5 \mathrm{~h}$ after the last injection. To avoid experimenter-dependent bias, brain were microdissected by a single investigator. Brains were rapidly removed from $\mathrm{CO}_{2}$-killed mice and placed in ice-cold slurry of $0.9 \%$ $\mathrm{NaCl}$. Rostro-caudal sections $(2 \mathrm{~mm})$ were quickly obtained on a brain tissue blocker. Four consecutive sections from Bregma +2.4 to -3.6 (Paxinos and Franklin, 2001) were transferred to RNAlater (Ambion Inc., Austin, TX) and microdissected. CC was dissected from the first two sections and included part of the prelimbic cortex. AMY was obtained from the third section and DG from the third and the fourth sections. Corpus callosum and anterior commissure were collected as a combined white matter (WM) sample. Samples were stored in RNAlater at $-80^{\circ} \mathrm{C}$.

\section{Microarray Samples}

Total RNA was extracted with Trizol (Invitrogen, Carlsbad, CA) and assessed by chromatography (Agilent Bioanalyzer, Santa Clara, CA). Average expert scoring number (RIN) was $8.48 \pm 0.03$ (mean \pm SEM), consistent with excellent RNA quality. Microarray samples were prepared according to the manufacturer's protocol (Affymetrix, Santa Clara, CA). In brief, $3 \mu \mathrm{g}$ of total RNA were reverse-transcribed and converted into double-stranded cDNA. A biotinylated complementary RNA (cRNA) was transcribed in vitro, using an RNA polymerase T7 promoter introduced during the reverse transcription. Fragmented labeled cRNA sample $(20 \mu \mathrm{g})$ was hybridized onto MOE430-2.0 microarrays. A high-resolution image of the hybridization pattern was obtained by laser scanning and stored in a raw file. Samples were randomly distributed at all experimental steps to avoid simultaneous processing of related samples. Probeset signal 
intensities were extracted with the Affymetrix Microarray GCOS software for assessment of quality control, and with the Robust Multi-array Average algorithm (Irizarry et al, 2003) for data analysis. Six arrays were retained per experimental group (Figure 1), representing a total of 84 arrays. Microarray quality control parameters (Mean \pm SEM) were as follows: noise (RawQ) $<5(1.83 \pm 0.05)$, background signal $<100$ (250 targeted intensity for array scaling; $52.5 \pm 1.3$ ), consistent number of genes detected as present $(57.2 \pm 0.1)$, consistent scaling factors $(1.29 \pm 0.06)$, actin and GAPDH $3^{\prime} / 5^{\prime}$ signal ratios $<3$ (ACT, $1.27 \pm 0.01$, GAPDH, $0.85 \pm 0.01$ ), and consistent detection of BioB/C spiked controls. Probesets with average signal intensity below 10 in all groups were considered at background level and were removed, leaving 25859 probesets for analysis.

\section{Microarray Data Analysis}

The goal of the analysis was to use profiles of expression over large groups of genes as an 'experimental assay' to investigate molecular effects. Therefore, thresholds for gene selection were kept at moderate stringencies $(P<0.05$, changes $>20 \%$ ) and no correction for multiple testing were applied. This approach potentially carries a high rate of false positives at the single gene level (see discussion), but is helpful when investigating broader effects over larger sets of genes, as previously applied by us and others to the characterization of brain function (Berton et al, 2006; Sibille et al, 2007b). Here, converging results across different treatments and concordance among treatments, behaviors, and gene expression were applied to identify UCMS and drug effects. For UCMS and UCMS + drug effects, ANOVA models with UCMS exposure and drug treatments as cofactors were fitted to all transcripts in each brain region, followed by two-group analyses based on the relevant questions (ie, UCMS effect, antidepressant effect, unpaired $t$-statistics). To assess antidepressant reversal of UCMS effect, we measured the extent by which drug treatments brought transcripts back to non-stressed levels, with ' $0 \%$ reversal' meaning that antidepressant had no effect on reversing the UCMS effects on that gene, and ' $100 \%$ reversal' meaning that gene transcript levels were back to control non-stressed control levels in UCMS-/drug-treated animals. Reversal was capped at $100 \%$ for genes whose antidepressant-related changes were in opposite directions from UCMS effects (Figure 5). Pearson correlation coefficients were used to measure similarities in expression profiles between areas and/or treatments using average $\log _{2}$-based ratio of changes.

\section{WM/GM ratios and Glial vs Neuronal Enrichment of Genes}

Ratios of transcript levels between gray matter (GM) and adjacent WM samples were calculated as estimates of relative glial to neuronal origins of transcripts for every gene within GM samples, as previously described (Sibille et al, 2008). The three brain areas were compared to a unique set of seven WM samples. On the basis of $\sim 25000$ expressed genes, the percentage of genes displaying neuronal enrichment, glial enrichment, or expressed in both cellular populations were assessed using a 1.5-fold level of enrichment. Results were used as reference values to compare 'expected' $v s$ observed distributions in groups of identified genes (see Table 1).

\section{Functional Classification of Genes}

Biological functions for lists of genes were annotated using the Database for Annotation, Visualization and Integrated Discovery (DAVID) tool (Dennis et al, 2003) that is freely available at http://david.abcc.ncifcrf.gov/. Over-representation of gene ontology (GO) terms was calculated using the default parameters of the 'functional annotation clustering' tool under the 'high' classification stringency set up. To reduce the redundancy of annotations, this tool groups together results in clusters of GO groups with similar annotations. $P$-values associated with each annotation are calculated with the Fisher's exact test.

\section{Real-Time Quantitative PCR}

cDNAs were obtained from the original RNA pool used for array analysis. PCR products were amplified in quadruplets on an Opticon real-time PCR machine (Bio-Rad, Hercules, CA), as previously described (Galfalvy et al, 2003). Primerdimers were assessed by amplifying primers without cDNA. Primers were retained if they produced no primer-dimers or non-specific signal after 35 cycles. Results were calculated as relative intensity compared to actin.

\section{RESULTS}

UCMS-Induced Changes in Physical State are Reversed by Chronic Exposure to Fluoxetine and SSR125543

Mice were submitted to a 7-week UCMS regimen or maintained under normal non-stressful control conditions and were administered daily from the third week with the vehicle, an effective (fluoxetine), or a putative antidepressant drug $\left(\mathrm{CRF}_{1}\right.$ antagonist SSR125543) (Figure 1). UCMS exposure resulted in a progressive deterioration of the coat state (Figure 2a), which became significant across groups at the beginning of the third week $(P<0.05)$, reached a plateau at the beginning of the fifth week and remained significantly elevated compared to control mice until the end of the experiment $(P<0.001)$. Fluoxetine-treated UCMS mice began to show a reversal of the coat state deterioration after 3 weeks of treatment (corresponding to the fifth week of UCMS). This improvement in coat state became significant at the beginning of the fourth week of treatment ( $P<0.01$, compared to UCMS vehicle mice), while fur scores of fluoxetine-treated mice were not different from control mice after 5 weeks of treatment $(P<0.001$, compared to UCMS/vehicle mice; $P>0.05$, compared to control mice).

Similarly, treatment with SSR125543 reversed the UCMSinduced deterioration of the coat state, but with a faster onset of action. The coat state of UCMS/SSR125543-treated mice did not further degrade during the first week of treatment and was significantly different from UCMS/vehicle mice after only 2 weeks of SSR 125543 exposure $(P<0.001)$. This improvement persisted until the end of the experiment $(P<0.001$, after 3,4 , and 5 weeks of treatment). The coat state of UCMS and SSR125543-treated mice had returned to 
normal condition after 3 weeks of treatment $(P>0.05$, compared to control mice). No changes in fur coat state were observed in control mice treated with vehicle, fluoxetine, or SSR125543.

UCMS treatment also induced significant differences in body weight changes (Figure $2 \mathrm{~b}$ ). Normal weight gain was reduced by UCMS starting in the fifth week of treatment $(P<0.05)$. The statistical significance of the reduced weight gain was strengthened in the last 2 weeks of the experiment $(P<0.001)$. Both fluoxetine and SSR125543 treatments partially blocked the UCMS-induced reduction of body weight gain in a significant manner in the last week of the experiment, corresponding to 5 weeks of drug treatment $(P<0.05$, compared to UCMS/vehicle mice). However, contrary to the effect on coat degradation, the effects of fluoxetine and SSR125543 on weight gain followed overlapping trajectories. Both compounds had no effect on body weight gain in non-stressed control mice.

Taken together, these results demonstrated a progressive effect of UCMS on physical aspects (fur coat degradation and reduced weight gain) that were reversed by chronic exposure to an effective (fluoxetine) or putative (SSR125543) antidepressant drug. Trajectories of drug reversals of UCMS effects demonstrated delayed onsets that paralleled the clinical time frame of the therapeutic effects of antidepressant treatments, although SSR125543 displayed a shorter delay of onset on the fur index compared to fluoxetine.

\section{UCMS-Induced Behavioral Changes are Reversed by Chronic Exposure to Fluoxetine and SSR125543}

UCMS exposure has been previously shown to induce a series of behavioral changes, including increased agonistic and anxiety-/depression-like (ie, 'emotion-related') behaviors that are reminiscent of symptoms of depression in human subjects (Mineur et al, 2003; Santarelli et al, 2003). Mice used in the investigation of UCMS-induced physical changes were not submitted to behavioral tests to preserve a non-stressed state in control mice for the microarray analyses and the real-time quantitative PCR (qPCR), thus results presented here were obtained in parallel cohorts of the same age and exposed to the same UCMS protocol.

An NSF test was performed after a 45 days of UCMS to assess putative alterations in emotion-related behavior due to UCMS application. A 12-h food deprived mouse was introduced in an arena in which a pellet of regular food is placed in the center. This test induces a conflicting motivation between the drive to eat the food pellet and the fear of venturing into the center of the arena. The latency to a

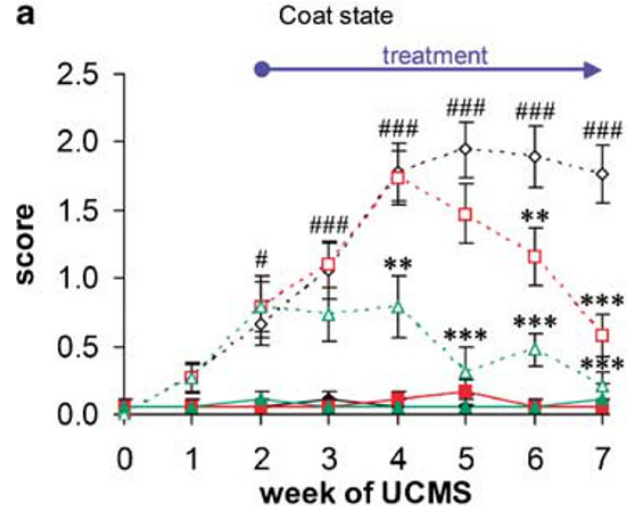

C

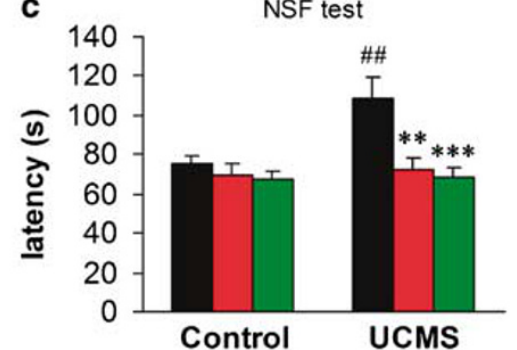

b

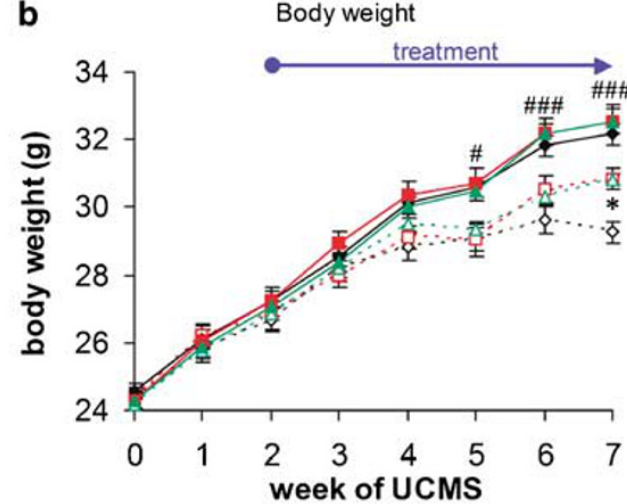

d

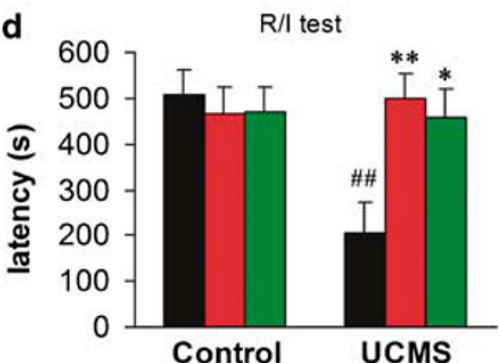

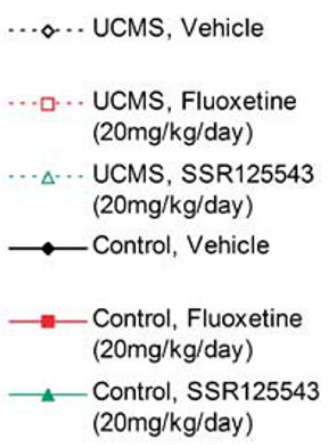

Figure 2 UCMS-induced changes in physical state and behavior are reversed by chronic treatment with fluoxetine or SSRI25543. (a) UCMS induced a significant deterioration of the coat state, as demonstrated by increasing coat state scores (see Materials and methods). Drug treatments initiated in the third week of UCMS exposure reversed this deterioration after 2 weeks of SSRI25543 treatment and after 4 weeks of fluoxetine treatment (ANOVA: environment, week I, $F_{1,104}=9.34, P<0.01$; weeks $2-7 F_{1,104}>35.06, P<0.00 I$; treatment, week $4, F_{2,104}=6.39, P<0.01$, weeks 5-7, $F_{2.104}>15.8$, $P<0.00$ I; environment $\times$ treatment: weeks $4-7, F_{2,104}>6.39, P<0.0$ I). (b) UCMS significantly disrupted the normal gain in body weight, starting at the fifth week of UCMS regimen. The progressive reversal of UCMS effect on weight gain by fluoxetine and SSRI 25543 exposure became significant in the last week of UCMS (ANOVA: environment, weeks 4-7, $F_{1,104}>10, P<0.001$; treatment, week $7, F_{2,104}=5.34, P<0.01$ ). $n=18-19$ mice per group. Post hoc Tukey test: ${ }^{\#} P<0.05$ and ${ }^{\# \# \#} P<0.00$ I for UCMS/vehicle mice vs control/vehicle mice; ${ }^{*} P<0.05$, $* * P<0.0$ I and $* * * P<0.00$ I for UCMS/treated group vs UCMS/ vehicle group. (c) The latency to begin eating in the NSF test was increased by UCMS. This effect was reversed by both fluoxetine and SSRI25543 treatments (ANOVA: environment, $F_{1,65}=5.79, P<0.05$; treatment, $F_{2,65}=8.49, P<0.00$ I; environment $\times$ treatment, $F_{2.65}=3.89, P<0.05$ ). $(d) \cup C M S$ induced a significant decrease in the latency to attack the intruder in the R/I test, while both fluoxetine and SSRI25543 treatment reversed this effect. (ANOVA: environment $\times$ treatment, $\left.F_{2,65}=4.75, P<0.05\right) . n=1 \mathrm{I}-12$ mice per group. Post hoc Tukey test: ${ }^{\# \#} P<0.0$ I for UCMS/vehicle mice vs control/ vehicle mice; $* P<0.05$, ${ }^{*} P<0.0$ I and ${ }^{*} * * P<0.00$ I for UCMS/drug-treated group vs UCMS/vehicle group. Data represent mean $\pm S E M$. 
chew the pellet was significantly altered (Figure 2c). UCMSexposed mice showed an increased latency compared to control mice $(P<0.01)$. Fluoxetine as well as SSR125543 significantly counteracted this UCMS-induced behavioral alteration $(P<0.01$ and 0.001 , respectively). Moreover, neither the UCMS procedure nor the drugs tested produced a significant change in the home food consumption during the 5 min following the NSF test (data not shown), suggesting that the feeding drive subsequent to a 12 -h deprivation was not different between experimental groups.

To evaluate changes in agonistic behavior, a R/I test was performed after a 50-day UCMS procedure. An intruder mouse was placed in the home cage of UCMS or control mice chronically treated with vehicle, fluoxetine, or SSR125543. The latency of the first attack and the number of attacks by the resident mouse were recorded. Significant differences were found for both measures (Figure 2d). UCMS exposure significantly reduced the latency $(P<0.01)$ and increased the number of attacks (data not shown; $P<0.05)$. Fluoxetine and SSR 125543 treatments completely reversed the UCMS-induced disruption in social encounter in latency (fluoxetine, $P<0.01$ and SSR125543, $P<0.05$ ) and in number of attacks (fluoxetine, $P<0.01$ and SSR125543, $P<0.05)$. Antidepressant treatments had no effect in control mice.

Taken together, these results demonstrated that in addition to inducing physical changes, UCMS induced a pattern of increased agonistic and emotion-related behaviors that were significantly reversed by chronic exposure to an effective (fluoxetine) or putative (SSR125543) antidepressant drug, thus corresponding to 'depressed' (UCMS syndrome) and 'recovered' (antidepressant-reversed) states in the UCMS model.

\section{The Gene Expression Correlates of a 'Depressive-Like' State Vary Across Corticolimbic Brain Regions}

To identify changes in gene expression correlating with UCMS and antidepressant treatments, we performed a microarray analysis at the 7-week time point, corresponding to the period of (1) established increase in fur coat index, (2) increased agonistic and emotion-related behaviors, and (3) complete reversal of both effects by fluoxetine or SSR125543 (Figure 1). Samples were obtained from the CC, AMY, and DG ( $n=6$ arrays per treatment and per brain area), as brain areas for which the human homologous areas participate in a corticolimbic network of mood regulation that is affected in depression (Mayberg, 1997; Seminowicz et al, 2004). RNA samples were processed on MOE430 2.0 arrays (Affymetrix Inc.), which interrogate the levels of $\sim 40000$ gene transcripts (see Materials and methods). As the analytical goal was to use profiles of expression over large groups of genes as an 'experimental assay' to measure UCMS and drug effects, thresholds for gene selection were kept at moderate stringencies for group comparisons $(P<0.05$ and changes greater than $20 \%$; see Materials and methods and Discussion). We have previously demonstrated the validity of this approach at characterizing robust and significant biological events in brain tissue (Sibille $e t a l$, 2007). Accordingly, the levels of 254 gene transcripts were significantly affected by UCMS in CC, 299 in AMY, and 166 in DG (Figure 3; Supplementary Tables S1-S3).

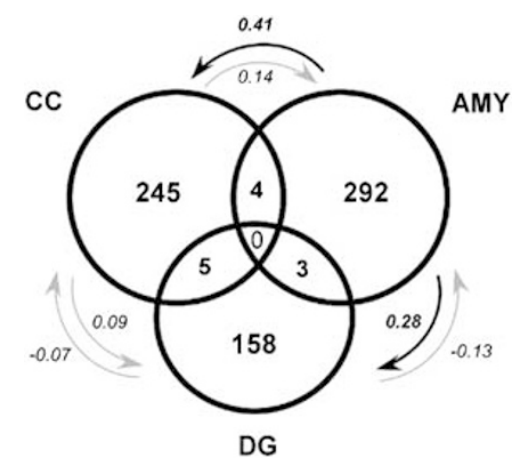

Figure 3 Corticolimbic brain region specificity of UCMS-induced changes in gene transcript levels. Venn diagram of transcript levels with significant UCMS effect in CC, AMY, and DG. Changes were mostly restricted to each of the three brain areas investigated, as little overlap was observed across areas. Arrows indicate directional correlations between changes in transcript levels for genes identified in one area (origin of arrow) and changes for the same genes in the other area (end of arrow). Italic values are Pearson coefficient factors $(r)$ of corresponding correlations. Bold italic values indicate significance $r$ values $\left(P<5 e^{-5}\right)$.

Very little overlap was observed across the three areas investigated, although transcript changes in AMY moderately, but significantly, predicted similar trends for the same transcripts in CC and DG (bold arrows in Figure 3, Pearson correlation $=0.41$ and 0.28 , respectively, $P$-values $<5 \times \mathrm{e}^{-5}$ ). On the other hand, UCMS-induced transcript changes in CC or DG did not predict corresponding changes or trends for the same transcripts in any other areas (gray arrows in Figure 3, Pearson correlations $P$-values $>0.05$ ). Independent real-time qPCR assessments of gene transcript levels significantly correlated with array results (Figure 4; $n=10$ genes, $r=0.72, P<0.01$ ), thus confirming the validity of the array measurements.

Taken together, these results suggested that the gene expression correlates of the UCMS-induced 'depressive-like' state were mostly brain region-specific within a corticolimbic network of mood regulation, although AMY changes moderately predicted similar trends for the same transcripts in $\mathrm{CC}$ and $\mathrm{DG}$.

\section{Fluoxetine and SSR125543 Reverse the Effect of UCMS on Gene Expression}

We next investigated whether the reversal of the UCMS physical and behavioral phenotypes by chronic antidepressant treatments also correlated with a reversal of the UCMS molecular phenotype. For each gene affected by UCMS, the percentage of reversal of UCMS effect by chronic fluoxetine or SSR125543 was calculated and gene-wise values were averaged for all UCMS-affected genes in each brain area (see Materials and methods). For instance, in CC, the chronic fluoxetine treatment reversed the effect of UCMS on gene expression by $\sim 75 \%$, meaning that the residual changes in transcript levels in the UCMS- and fluoxetine-treated group represented on average only $\sim 25 \%$ of the full UCMS effect (Figure 5a, middle panel). SSR 125543 reversed only $\sim 44 \%$ of the UCMS molecular profile in CC (Figure 5a, lower panel). In AMY, both drug treatments reversed the UCMS effect to a large extent ( $>70 \%$; Figure $5 \mathrm{~b}$, middle and lower 
panels). In contrast, these effects were weaker in DG, reaching only 28 and 39\% reversal for fluoxetine and SSR125543, respectively (Figure 5c, middle and lower panels) Tables S1-S3.

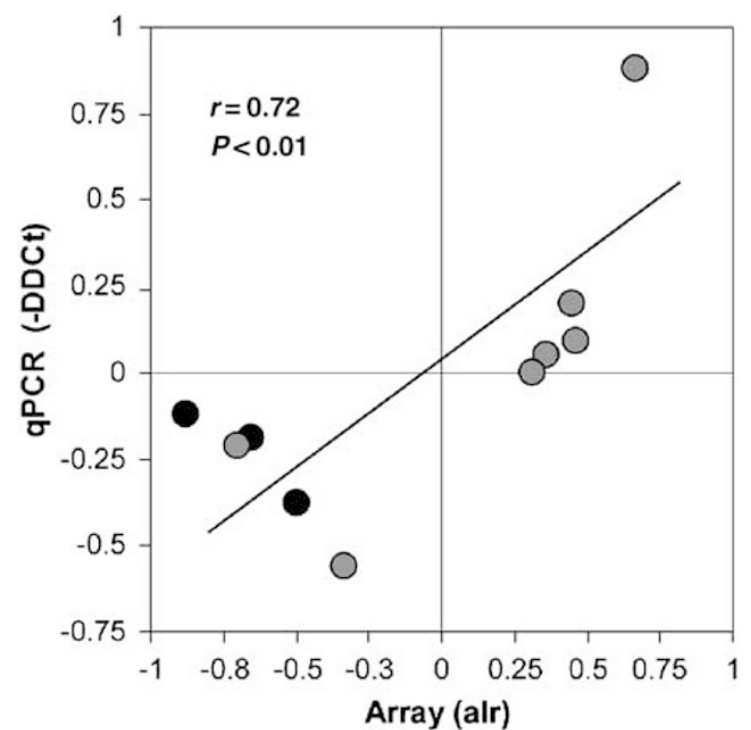

Figure 4 Validation of array results by independent qPCR measurements. Ten genes with significant differences in AMY were independently assessed by qPCR (Mobp, Cnpl, Enpp2, Cpne9, Pitpncl, Rph3a, Dgkg, Gabraa2, Kctd 12, and Arhgap6). Black dots represent oligodendrocyterelated genes (Mobp, Cnpl, and Enpp2) (see Table 2). r, Pearson correlation factor; Alr, average $\log _{2}$ of (UCMS/control) expression ratio; -DDCt represent differences in PCR cycle thresholds between UCMS and control samples, which are equivalent to $\log _{2}$ values of ratios.
Thus, antidepressant treatments reversed the effect of UCMS on altered gene expression, although the extent of reversal varied across areas and treatments according to the following order: $\left(\mathrm{AMY}_{\text {fluoxetine }}=\mathrm{AMY}_{\mathrm{SSR} 125543}=\right.$ $\mathrm{CC}_{\text {fluoxetine }}$ ) $>\mathrm{CC}_{\mathrm{SSR} 125543} \geqslant \mathrm{DG}_{\mathrm{SSR} 125543}>\mathrm{DG}_{\text {fluoxetine. }}$ Overall, AMY displayed highest and most consistent antidepressant reversals of UCMS effects.

\section{Fluoxetine and SSR125543 Treatments Induce Additional and Very Similar Transcriptome Effects in a Brain Region-Specific Manner}

Beyond reversing the molecular correlates of UCMS, fluoxetine and SSR125543 treatments affected the expression levels of very large numbers of additional genes (fluoxetine: 2540 genes in CC, 640 genes in AMY, and 294 genes in DG; SSR125543, 562 genes in CC, 507 genes in AMY, and 122 genes in DG; Table S4), yielding a pool of $\sim 3600$ genes affected by antidepressant treatments in any of the three brain areas investigated. Interestingly, very few genes were affected by the same drug across brain areas ( $<10 \%$ of the fluoxetine-related gene pools and $<2 \%$ of the SSR125543 pools), while much larger number of genes were similarly affected by the two different drug treatments within each brain area (48\% in CC, $25 \%$ in AMY, and $18 \%$ in DG) (see Supplementary Table S4). Accordingly, transcript changes displayed high correlations between fluoxetine and SSR125543 effects within each brain area (Figure 6a), but low correlations for the effects of the same treatment across two different brain areas (Figure 6b).
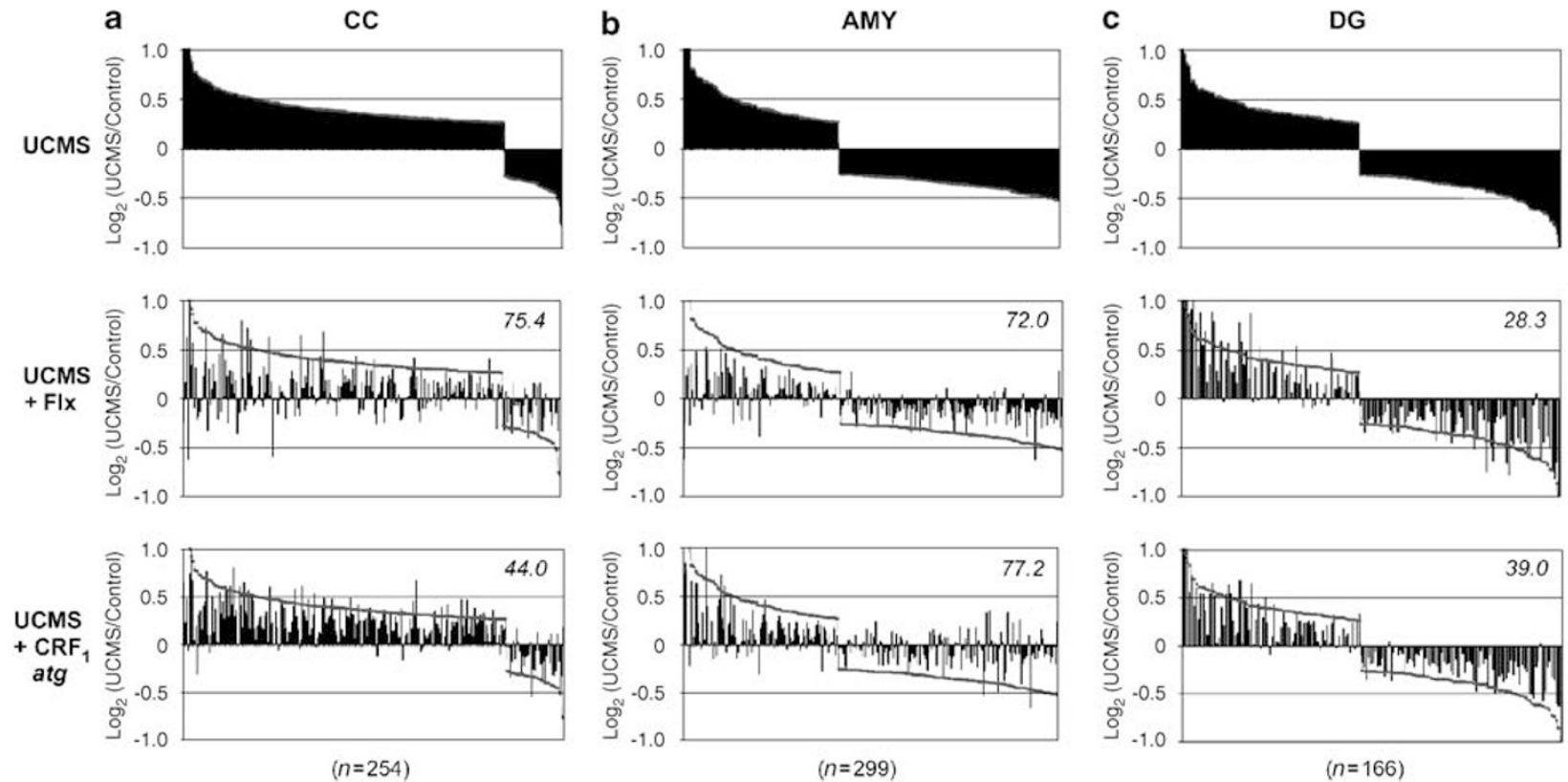

Figure 5 Differential reversal of the UCMS gene expression profiles by antidepressant treatments. Profiles of changes in gene transcript levels for UCMSaffected genes (top panels) and after reversal by antidepressant treatments (middle and low panels) in CC (a), AMY (b), and DG (c). Selected gene groups are from Figure 3. Top panels: UCMS-affected genes are organized by the magnitude of their changes in transcript levels along the $x$ axis and form a continuous profile with a pre-determined 20\% effect cutoff value (see Materials and methods). Vertical bars indicate the amplitude of changes (log 2 of UCMS/control expression ratio). Middle and low panels: vertical bars indicate the relative transcript levels for the same UCMS-affected genes after chronic fluoxetine or SSRI 25543 treatment. Note that the profiles have shifted away from UCMS levels (black contour line), back toward control 'no change' levels $\left(\log _{2} r \sim 0\right)$. The average percentage of drug reversal of the UCMS molecular profile is indicated per brain area and drug treatment. Flx, fluoxetine; CRF, atg, SSRI 25543. 
a

Flx vs $\mathrm{CRF}_{1}$ atg within areas $x$

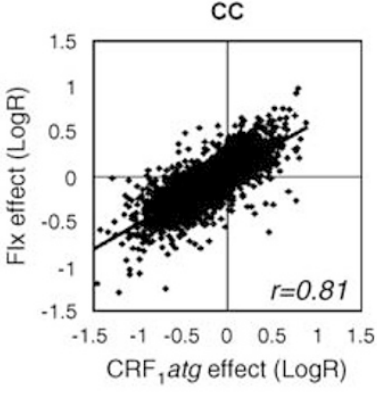

b
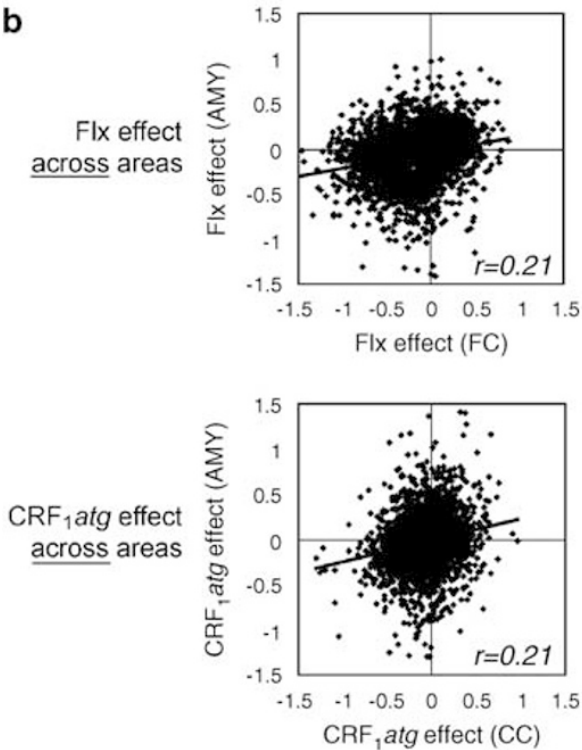
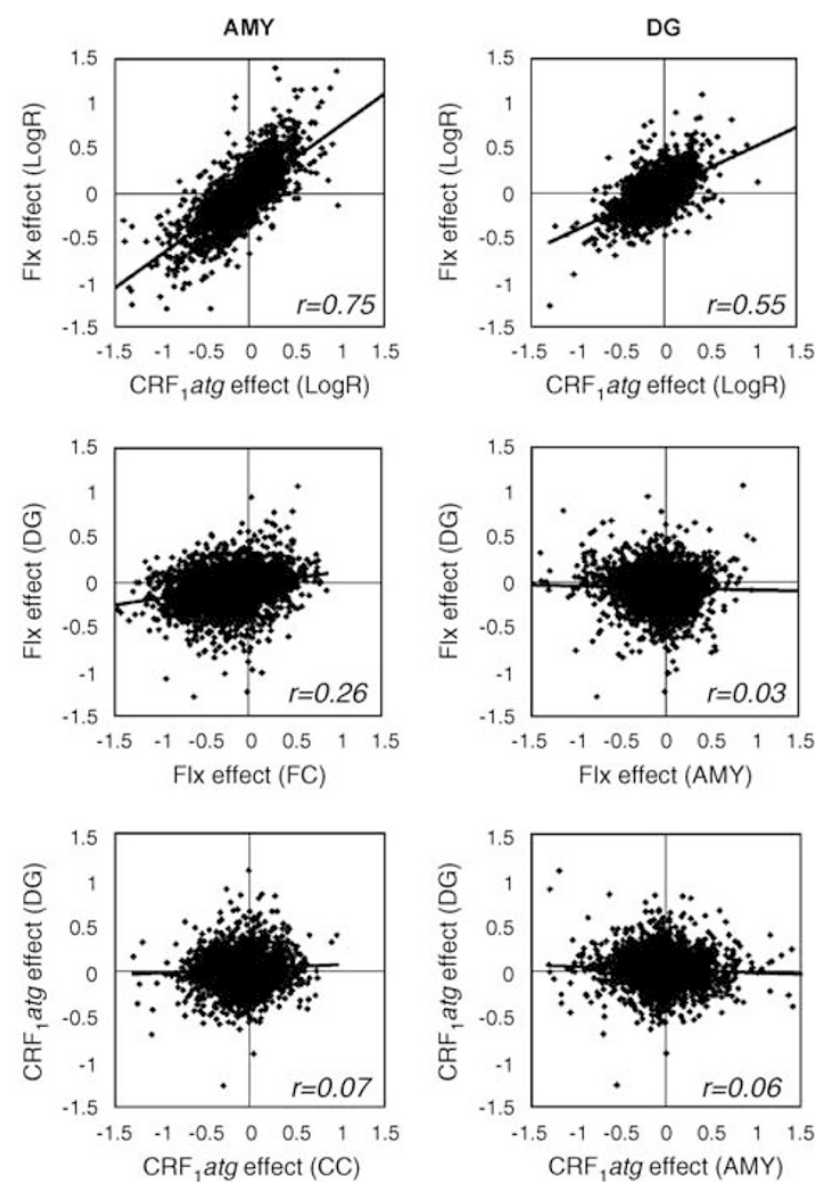

Figure 6 The transcriptome effects of two different drug treatments are more similar within brain areas, compared to the effect of each individual treatment across areas. For a comprehensive overview, the effects of antidepressant treatments on gene transcript levels are depicted for the pool of $\sim 3600$ genes that were affected by either treatment in any of the three areas (see text). Similar results were observed with more restricted pools of genes based on single drug effect or within a single brain area (not shown). (a) High correlations (0.55-0.8I) and high graph slopes for linear fit (0.46-0.72, black bars) were observed between the effects of two antidepressant treatments on gene transcript levels within a brain region, indicating high similarity of molecular impacts for the two different drug treatments. (b) Low correlations ( -0.03 to 0.26$)$ combined with low graph slopes $(-0.03$ to 0.17$)$ reveal poor similarities in the molecular impact of a same drug treatment across any two brain areas investigated. $x-y$ axes values are $\log _{2}(U C M S$ antidepressant-treated/control) for the respective drug treatment. Solid bars indicate trend lines. $r$, Pearson correlation coefficients; Flx, fluoxetine; CRF, atg, SSRI25543.

These results revealed that, in addition to reversing the effect of UCMS, antidepressant treatments affected the transcript levels of large numbers of genes, and that the transcriptional profiles of the two different drug treatments were more similar within a brain area, compared to the effect of the same treatment across areas, thus demonstrating that the molecular impacts of fluoxetine and SSR 125543 treatments were mostly determined by the brain area, rather than by their molecular targets (see Discussion).

\section{Transcriptome Effects of Fluoxetine and SSR125543 Treatments are State-Dependent in CC}

The large-scale effects of antidepressant treatments have been mostly characterized in normal or control animals and it is not known whether results apply to depressivelike states. Here, we directly addressed this question by comparing the effects of chronic fluoxetine and SSR 125543 exposure on CC gene expression in control and in UCMStreated mice. Results indicated that larger number of transcript changes was induced by both drug treatments in control mice (3221 genes for fluoxetine and 3004 genes for SSR125543; Table S5) compared to UCMS-treated mice (2540 genes for fluoxetine and 562 genes for SSR125543). Similar to the results in UCMS-treated mice, the molecular impacts of the two different drug treatments were strikingly similar in control mice (Figure $7 \mathrm{a}, r=0.94$, slope $=0.90$ ). On the other hand, comparing the effects of fluoxetine or SSR125543 between control and UCMS-treated groups revealed much lower correlations $(r=0.22$ and 0.46$)$ and greatly reduced amplitudes of altered transcript levels (Figure $7 \mathrm{~b}$, slopes $=0.11-0.19$ ), denoting a poor conservation of drug effect between control and UCMS states. The converse analysis of identifying genes that were modulated by drug treatments in UCMS-treated mice and comparing the extent of transcript changes for those genes in drugtreated control mice yielded very comparable results ( $r=0.24-0.30$, slope $=0.26-29$; not shown $)$, thus confirming the low conservation of drug treatment effects between control and UCMS states.

Another notable difference was the magnitude of effects on gene transcript levels across states and treatments. 
Fluoxetine and SSR125543 each affected $\sim 12 \%$ of all genes with detectable expression in control animals, but only 9.8 and $2.2 \%$ in UCMS-treated states for fluoxetine and SSR125543, respectively. In comparison, less than $1 \%$ of the genes were affected by UCMS alone.

Taken together, these results revealed that, in addition to being brain area-dependent, the molecular correlates of antidepressant treatments were state-dependent in CC, as demonstrated by low similarities of drug-induced transcript profiles between UCMS and control states. Our results also revealed differences in drug-induced gene expression plasticity, with fewer genes affected by UCMS alone, and according to the following order of magnitude: UCMS $<$ drugs in UCMS $<$ drugs in controls.

Correlations between Gene Expression, UCMS Behavior and Antidepressant Treatments Identify Candidate Genes for Mood Regulation and/or Genes with Therapeutic Potential

On the basis of patterns of altered transcript levels, genes were classified according to their potential contribution to the expression of the UCMS phenotype and/or to the reversal of that phenotype (Figure 8). Specifically, we focused on two categories of genes of interest: (1) 'mooddependent and therapeutic' genes, as affected in opposite directions by UCMS and antidepressant treatments, and thus matching the behavioral phenotype, and (2) 'therapeutic-only' genes that were not affected by UCMS, but that displayed significant transcript changes after both antidepressant exposures in UCMS-treated mice (Figure 8). The assumption of 'therapeutic-only' potential was that a reversal of the UCMS phenotype may occur through biological pathways that are independent of UCMS effects. For both categories, a positive antidepressant effect had to correspond to significant and similar effects after both drug treatments, reflecting the similar reversal of physical and behavioral UCMS effects by fluoxetine and SSR125543.

A total of 768 putative 'mood-dependent and therapeutic' or 'therapeutic-only' genes were identified (Table 2 and Supplementary Tables S6-S11). Consistent with the previously described region-specific effects, gene selections displayed very limited overlap across brain areas. In AMY, genes of interest (Figure 8) were for the most part in the 'mooddependent and therapeutic' gene category $(n=241)$ compared to 'therapeutic-only' genes $(n=82)$, reflecting the extensive and uniform antidepressant reversal of UCMS effects by both treatments (see Figure 5b; ie, gene transcripts mostly tracked both UCMS and antidepressant effects). In CC, the discrepancy in the molecular impact of antidepressants (Figure 5a; $75.4 \%$ fluoxetine and $44.0 \%$ SSR 125543 reversals of UCMS effects) translated in fewer genes with 'mood-dependent and therapeutic' profiles $(n=146)$ and more genes with potential 'therapeutic-only' involvement $(n=238)$. In contrast, much fewer genes of interest were identified in DG (62 and 19, respectively), due to the combined low UCMS effect, moderate antidepressant reversal, and overall lower molecular impact of antidepressant treatments (see next, Table 2, and Supplementary Tables S6-S11).

In summary, correlations between altered gene expression, UCMS behavior, and response to antidepressant treatments identified brain region-specific candidate genes for mood regulation and/or genes with therapeutic potential, with AMY displaying the highest content of genes with potential dual contribution to the UCMS phenotype and therapeutic antidepressant reversal.

\section{Identified Gene Transcripts Suggest Different Oligodendroglial and Neuronal Molecular Phenotypes in Correlation with UCMS and Antidepressant Treatment in AMY}

We have previously shown that array data from adjacent WM samples can be used to generate WM/GM ratios that are specific for each gene (Erraji-BenChekroun et al, 2005; Sibille et al, 2008). WM/GM ratios can be used as estimates of gene transcript enrichment either in glia (WM/GM $>1.5$ ), neurons $(\mathrm{WM} / \mathrm{GM}<-1.5)$, or both cellular population $(-1.5<\mathrm{WM} / \mathrm{GM}<1.5)$ and provide a wider view of overall patterns relating to glial and neuronal functions (Sibille et al, 2008). For instance, displaying UCMS-affected genes according to the extent of their transcript changes (up and
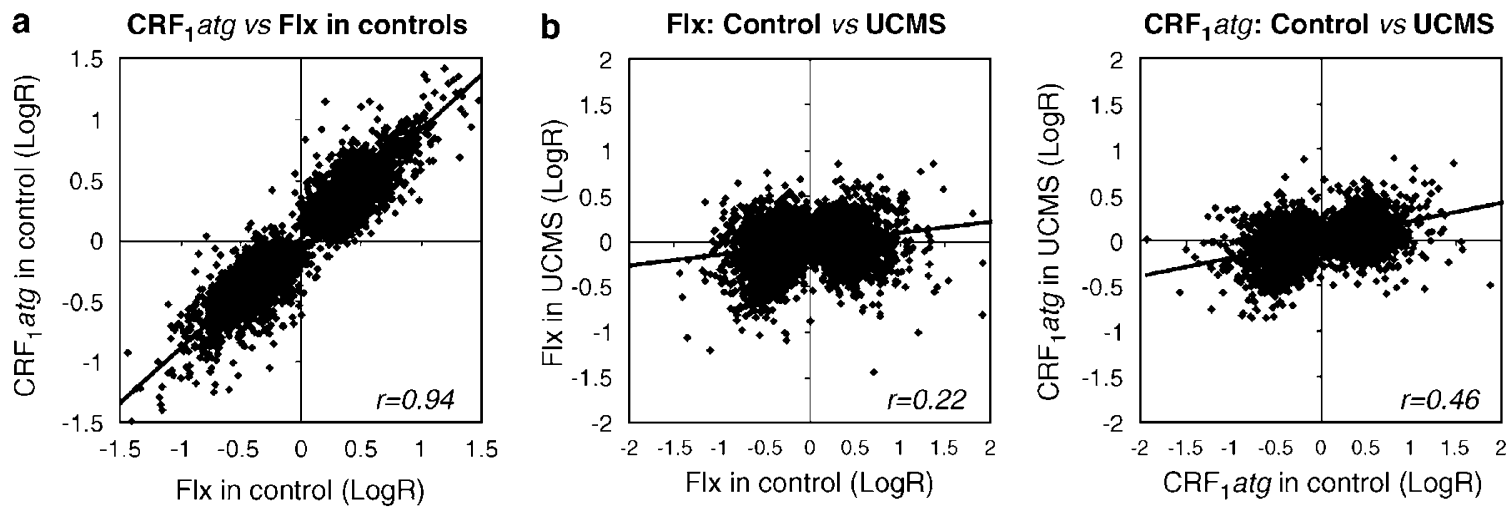

Figure 7 State-dependent effects of fluoxetine and SSRI25543 treatments in CC. (a) Chronic drug treatments induced extensive and highly similar transcriptome changes in control non-stressed mice. In total, 3221 genes were significantly affected by fluoxetine and 3004 genes for fluoxetine or SSR I 25543 with $\sim 60 \%$ of genes significantly affected by both treatments (see Supplementary Information). The correlation graph includes a total of 4305 antidepressant-affected genes $(r=0.94$, slope $=0.90)$. (b) Lower correlations $(r=0.22-0.46)$ and much reduced graph slopes $(0.1$ I -0.19$)$ suggest that the same drug has different effects in control and UCMS-treated mice. $x-y$ axes values are $\log _{2}$ (UCMS antidepressant-treated/control) for the respective drug treatment; $r$, Pearson correlation coefficients; Flx, fluoxetine; CRF,atg, SSRI 25543. 


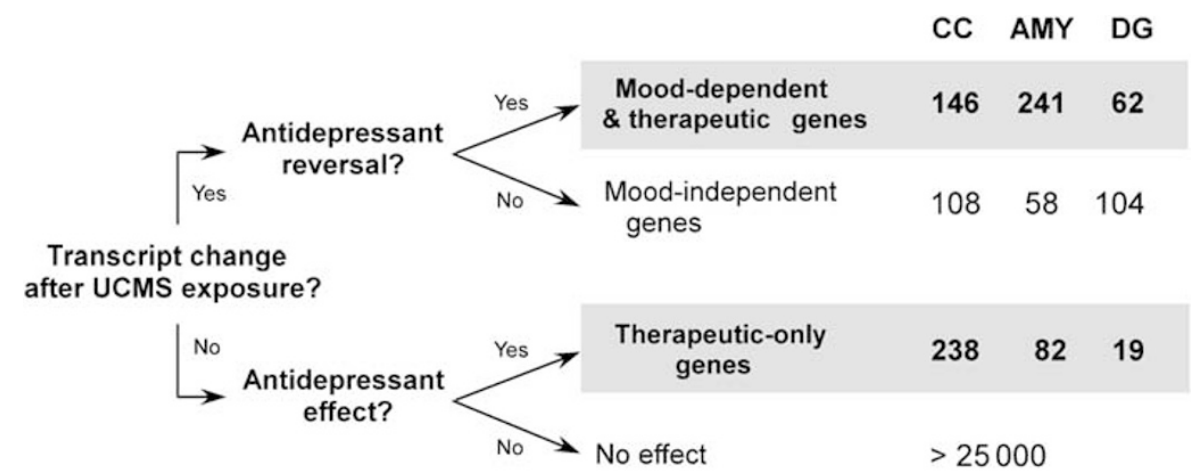

Figure 8 Patterns of UCMS behavior/gene expression/antidepressant effect identified candidate genes for involvement in mood regulation or with potential for therapeutic applications. For positive antidepressant reversal or for antidepressant effect, genes had to be significantly and similarly affected by fluoxetine and SSRI25543 treatments.

Table I Proportional representations (\%) of transcripts with enriched glial or neuronal origin within groups of identified genes

Cellular enrichment of gene transcripts

\begin{tabular}{|c|c|c|c|c|}
\hline & \# Genes & $\begin{array}{c}\sim \\
\text { Neuronal } \\
(\%)\end{array}$ & $\begin{array}{c}\text { Both } \\
\text { (\%) }\end{array}$ & $\begin{array}{c}\sim \text { Glial } \\
(\%)\end{array}$ \\
\hline CC & & $24 *$ & $56 *$ & $20 *$ \\
\hline 'Mood and therapeutic' & 146 & 19 & 53 & 28 \\
\hline 'Therapeutic only' & 238 & 21 & 59 & 20 \\
\hline AMY & & $23 *$ & $57 *$ & $20 *$ \\
\hline 'Mood and therapeutic' & 241 & 23 & 38 & 39 \\
\hline 'Therapeutic only' & 82 & 46 & 37 & 17 \\
\hline DG & & $23 *$ & $53 *$ & $24 *$ \\
\hline 'Mood and therapeutic' & 62 & 23 & 48 & 29 \\
\hline 'Therapeutic only' & 19 & 11 & 36 & 53 \\
\hline
\end{tabular}

'\# genes' indicates the number of genes identified in the respective category. Italic values with (*) indicate expected proportions of glial- and neuronalenriched genes (see Materials and methods). Gene categories are described in the text. Bold numbers represent values that are significantly different from expected proportions ( $\chi^{2}$ tests, $P<0.05$ adjusted for multiple comparisons). Differences from expected proportions in 'therapeutic only' genes were not significant in DG, due to the low number of genes within that category.

down arrows in Figure 9) and based on their estimated cellular origin of transcripts (yellow, gray, or black bars in Figure 9) clearly identified predominantly downregulated glial-enriched genes in AMY (Figure 9a), as visually represented by the overall yellow color in the lower portion of the central panel. Within our categories of genes of interest (Figure 8), statistical assessments confirmed that AMY glialenriched genes were significantly over-represented in the 'mood and therapeutic' gene category, while neuronalenriched genes were more numerous in the 'therapeuticonly' gene categories (Table 1). The CC and DG 'mood and therapeutic' and 'therapeutic-only' gene pools displayed proportions of glial- or neuronal-enriched gene transcripts that did not differ greatly from their expected proportions
(Table 1), with the exception of a slight over-representation of glial-enriched genes in correlation with mood levels in CC.

While caution should be applied for identification of single genes in array studies under medium statistical stringency (see Discussion), looking at the convergence of single genes (Table 2) and at over-representation of biological functions within the lists of identified genes (Table 3) revealed complex sets of changes involving numerous cellular functions (receptors, signaling, transcription, metabolism, structural components, etc.). Summarizing this information, three important themes emerged. First, consistent with brain region-specific effects of UCMS and antidepressant treatments, none of the selected genes were identified across regions. Second, genes coding for several components of neurotransmitter systems (GABA, glutamate, and peptides), signal-transduction pathways (PKC, PLC, and MAPK) and second messenger systems (cAMP) previously associated with altered mood and/or antidepressant treatments were identified here (underlined in Table 2), were over-represented in AMY (20 genes out of $31, P=0.005)$. Third, numerous oligodendrocyte markers were exclusively downregulated within the 'mood and therapeutic' gene category in AMY (Mobp, Edg2, Gsn, Cnp1, Gpr37, Pllp, Enpp2, Pmp22, Mpzl1, and Plp1; bold in Table 2; 'myelin' functional cluster in Table 3; black dots in Figure 4), thus strongly suggesting an antidepressant-sensitive oligodendrocyte-related phenotype in the AMY of UCMS-treated mice (see Discussion).

Taken together, these results revealed different and complex molecular phenotypes in correlation with UCMS and antidepressant treatments in the three brain regions investigated, but also identified the AMY as a crucial area of interest, with a neuronal molecular pathology affecting previously identified candidate neurotransmitter systems, and a glial phenotype focused on UCMS-downregulated markers of oligodendrocyte structure and function, in reminiscence of prior reports in the AMY (Hamidi et al, 2004) and in nearby cortical regions (Aston et al, 2005) of human depressed subjects. It is important to note that while our analytical approach relied on the cumulative effects across large group of genes to identify robust region- and state-dependent effects of UCMS and antidepressants, results for single genes should be considered in the context 
Table 2 Identified genes (selected) corresponding to 'mood' and/or 'therapeutic' gene categories in the three brain regions investigated

\begin{tabular}{|c|c|c|c|c|c|}
\hline Gene category & $\begin{array}{l}\text { Direction of } \\
\text { transcript } \\
\text { changes }\end{array}$ & \# Genes & $\begin{array}{l}\text { Neuronal-enriched (\# genes) gene } \\
\text { codes }\end{array}$ & $\begin{array}{l}\text { Neuronal and glial expressed } \\
\text { (\# genes) gene codes }\end{array}$ & Glial-enriched (\# genes) gene codes \\
\hline \multicolumn{6}{|l|}{ CC } \\
\hline \multirow[t]{2}{*}{$\begin{array}{l}\text { Mood and } \\
\text { therapeutic }\end{array}$} & UCMS $\uparrow \mathrm{AD} \downarrow$ & 121 & $\begin{array}{l}\text { (I8) Nrld2, Ankdl3c, Nanos I, Smoc2, Phfl7, } \\
\text { Pik3cd, Pclo, Acvr2a, Atpifl, Rragd, Thtpa, } \\
\text { Zh×3 ... }\end{array}$ & $\begin{array}{l}\text { (66) AR, Gspt2, Vps I 3a, Agpat6, Commd2, } \\
\text { Yipf5, ccdc84, Slc24a4, Pcdh 17, Tbc Id8, Lyst, } \\
\text { Toak3, Spen, Dnajb4, Auts2, Ints8, Stk4, } \\
\text { Atp IOa, Txnrd I, Dhodh, Cryl, Makpb I, } \\
\text { Exosc I, Lix II, MtrflI, Hexim I, FzdI, Stx I8, } \\
\text { Bcl9, Ccnd2, Suclg2, Siah2, Ift80, Ube2z, Evl, } \\
\text { P4ha2 ... }\end{array}$ & $\begin{array}{l}\text { (37) Ubie, Asph, Cth, Slc3 Ia2, Wdfyl, E2f5, } \\
\text { CCnd2, Dbt, Plch I, Osbpl9, Scn7a, Cklf, } \\
\text { Rps6ka5, Irf2bp2, Husl, Fcho I, Oprm I, } \\
\text { Gpsm2, Rbm3, Hist2h3cl, Hspalb, Tsnax, } \\
\text { Pdgfc, Tencl ... }\end{array}$ \\
\hline & UCMS $\downarrow \mathrm{AD} \uparrow$ & 25 & (5) Ddit41, Nptx2, Add2, Cast ... & $\begin{array}{l}\text { (I3) Bcll Ia, Cstad, Ptpra, Hlcs, Sema5b, } \\
\text { Grin2c, Ktcdl } 5 \text {... }\end{array}$ & (7) Ktcd 15, CaldI, Rcn3, Filip I ... \\
\hline \multirow[t]{2}{*}{$\begin{array}{l}\text { Therapeutic- } \\
\text { only }\end{array}$} & $\mathrm{UCMS} \leftrightarrow \mathrm{AD} \downarrow$ & 194 & $\begin{array}{l}\text { (45) Ovol2, Exph5, Npas4, Itga8, Cbln4, Tbrl, } \\
\text { Cxxc4, Cxadr, Ints8, St6gal2, Neurod6, } \\
\text { Ube3a, Grm8, Mmp 16, ENtp7, MapkIO, } \\
\text { Slc I9a2, RunxItI, Synj2, Per2, UbelI2, Klf4, } \\
\text { Ccnc, Hmgcr ... }\end{array}$ & 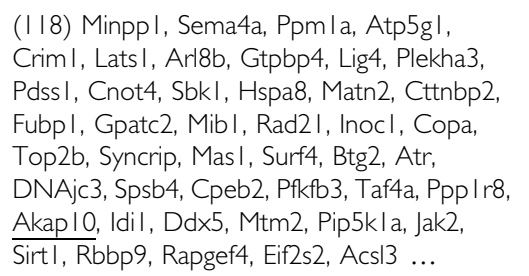 & $\begin{array}{l}\text { (31) Tob2, Nr2c2, Sfrs I, Sp3, Sptcl2, Idh I, } \\
\text { Gprk5, Gpt2, Dlg5, Map4k5, Elf2, Maml I, } \\
\text { Qser1, Gabrgl, InsmI, LrchI, Mettl7a, } \\
\text { Hspa Ib, Pkp2, Cacnb2, Prkcd ... }\end{array}$ \\
\hline & $\mathrm{UCMS} \leftrightarrow \mathrm{AD} \uparrow$ & 44 & $\begin{array}{l}\text { (I I) Pvalb, Nell2, Rab6b, Ntrk3, Usp7, Fndc5, } \\
\text { Rtn I ... }\end{array}$ & $\begin{array}{l}\text { (23) Plekha5, Acp I, Arhgef4, Commd5, } \\
\text { Endog, Gcap I4, Scn4b, Slc25a5, GdpdI, } \\
\text { Gpr I72b, Dagl, Jaridla, Psma4, Nfib, Fance, } \\
\text { Ntngl ... }\end{array}$ & $\begin{array}{l}\text { (10) Pcdh9, Slc26al I, EndodI, Ppr|rl6b, } \\
\text { Ednrb, WnkI, SI00a I6, Dbndd2 ... }\end{array}$ \\
\hline \multirow[t]{2}{*}{$\begin{array}{l}\text { Mood and } \\
\text { therapeutic }\end{array}$} & UCMS $\uparrow A D \downarrow$ & 82 & $\begin{array}{l}\text { (40) Igfbp3, Per2, Alkbh8, Nrip I, Grin2b, } \\
\text { Akap5, Dgkg, Atp I Ic, Kitl, Scn3a, Map2k6, } \\
\text { Cys I, Socs2, Srpk2, Ankrd43, Arhgap6, Npylr, } \\
\text { Npy2r, Cacnb2, Gda, Mchrl, Camk2d, Grin3a, } \\
\text { Kcnd3, Gprl78 ... }\end{array}$ & $\begin{array}{l}\text { (39) Katnal, Igf|r, Nfiib, Ccdc43, Chsyl, } \\
\text { Sec24d, Nfix, Josd3, Jun, Matn2, Cttnbp2, } \\
\text { Mgrn I, Peli I, NptxI, KctdI2, Taok3, Gabra2, } \\
\text { Ttc26, Nrg I ... }\end{array}$ & (3) Trp53bp2, Mett17a \\
\hline & UCMS $\downarrow \mathrm{AD} \uparrow$ & 195 & $\begin{array}{l}\text { (15) MppedI, Accn4, Prss I2, Elmol, Npy, } \\
\text { Sorcs3, Cckbr, Cutl2 ... }\end{array}$ & $\begin{array}{l}\text { (5I) Nuprl, Pdia5, Gpcl, Cxcl4, Itm2a, KIf7, } \\
\text { Adcy2, Aifl, Incenp, Diras2, Chrdl I, Hapln I, } \\
\text { Gnb4, Scotin, EgrI, LynxI, Igsf2 I, Gal3st3, } \\
\text { Rgs6, Rph3aPpp Irl6a, Rbbp4, Scubel ... }\end{array}$ & $\begin{array}{l}\text { (I44) Litaf, Mobp, Edg2, Adamts4, Ptprd, } \\
\text { Arhgap25, Trf, Gsn, Gpr37, Cdc42ep2, } \\
\text { Efhd I, Rhog, Cnp I, Itfg3, Apod, Gng I I, PIlp, } \\
\text { Bcas I, Enpp2, Dusp I6, Cryab, Pmp22, } \\
\text { Qdpr, Mpzl I, Rab3I, PIp I, Aga, Rgs3, Car2, } \\
\text { Rorb, LampI, Lgals, I, Prom I, Rtkn, Cpne9, } \\
\text { Grm3, Ifit2, P2rx4, Hip Ir, Plcd I, Slco2b I, Bok, } \\
\text { Dusp3, StardIO, Pgm I, Tinf2, Zyx ... }\end{array}$ \\
\hline \multirow[t]{2}{*}{$\begin{array}{l}\text { Therapeutic- } \\
\text { only }\end{array}$} & $\mathrm{UCMS} \leftrightarrow \mathrm{AD} \downarrow$ & 62 & $\begin{array}{l}\text { (36) Rpa2, Ap Igl, Slk, Pcdh20, Efnb2, Phip, } \\
\text { Pde7a, Rgmb, Arl5a, Ryr2, Timp2, Grin2a, } \\
\text { Smad3, Kcnj9, Apafl, Nptxr ... }\end{array}$ & $\begin{array}{l}\text { (22) TribI, Uspl, Tiparp, GPRI72b, Torlb, } \\
\text { Bzw2, Itpkc, Kpnb I, Pigh, Cpd, Htr4, SgplI, } \\
\text { Mycll ... }\end{array}$ & (4) Spa I7, Ss 18 , Slco3al \\
\hline & $\mathrm{UCMS} \leftrightarrow \mathrm{AD} \uparrow$ & 20 & (2) Cpne5 & (8) Gfpt2, Fxn, Dxd42, Trio, Adam23, Xrcc2, & (I0)Gja I2, Tmccl, VanglI, Rela, Slc22a8 \\
\hline
\end{tabular}


of the moderate statistical stringencies applied (see Discussion). Specifically, we make available tables of results as supplements so that individual results can be compared and validated across studies.

\section{DISCUSSION}

To gain insight into brain function during mood regulation, we investigated correlations between behavioral and molecular changes in the UCMS rodent model of depression and of antidepressant reversal. We confirm the validity of UCMS at inducing robust physical and behavioral changes that are reminiscent of a depressivelike state in mice, and show that both symptom dimensions are reversed by chronic exposure to an effective (fluoxetine) or a putative (SSR125543, $\mathrm{CRF}_{1}$ antagonist) antidepressant drug. Measuring changes in large-scale gene expression as an indirect assessment of brain function, we report that the molecular correlates of UCMS and antidepressant treatments differ across areas of a corticolimbic network of mood regulation (CC, AMY, and DG), and that two antidepressant treatments induced very similar transcriptome changes within areas, despite targeting different biological systems (brain regionspecific antidepressant effects). We also demonstrate that the effects of antidepressant treatments vary greatly depending on whether treated animals are in a control or 'depressed' state (state-dependent changes).

\section{Physical and Behavioral Changes Affected by the UCMS Model of Depression and Antidepressant Treatment}

UCMS exposure elicits a syndrome with a range of phenotypes that are analogous to symptoms of depression, including low stress coping and anxiety-/depression-like behaviors (Santarelli et al, 2003; Ducottet et al, 2004; Yalcin et al, 2005), decreased reward function (Pothion et al, 2004), increased glucocorticoid levels (Ayensu et al, 1995; Banasr et al, 2007; Li et al, 2007) and decreased hippocampal cell proliferation and neurogenesis (Alonso et al, 2004). Here, mice subjected to a 7-week UCMS paradigm developed a progressive deterioration of their coat state, a decline in weight gain, an exaggerated emotional reactivity in the NSF test, and social disturbances in the R/I test (Figure 2). Chronic administration of fluoxetine reversed these UCMS-induced deficits after 4 (coat state, NSF test) to 5 weeks (body weight, R/I test) of treatment. Likewise, SSR125543 showed antidepressantlike properties, as this compound counteracted all UCMSrelated effects, including with a faster onset of improvement in the quality of the coat state (after 2 weeks of treatment) compared to fluoxetine (3-4 weeks of treatment). Overall, antidepressant reversals of UCMS effects occurred in a time course that paralleled therapeutic improvements in depressed subjects, thus emphasizing UCMS as a valid model for investigating depressive pathophysiology and mechanisms of antidepressant reversal. Indeed, UCMS fulfills several criteria for a valid model of depression, including: (1) good face validity (close ethological counterpart for emotion-related and 
CC

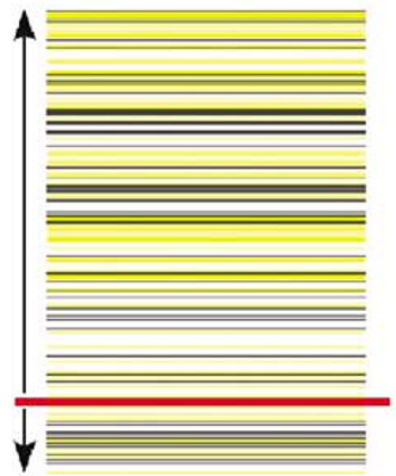

AMY

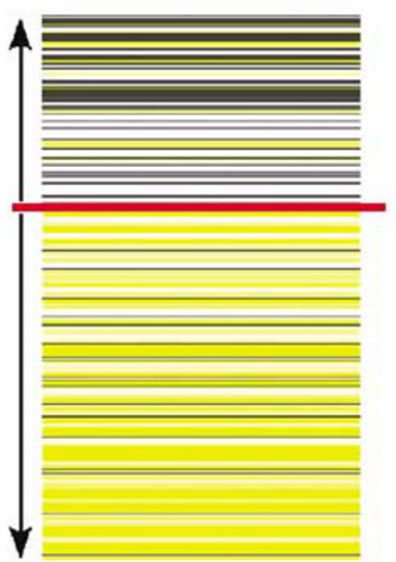

DG

Transcript origin:

$\sim$ glial

$\sim$ neuronal

woth

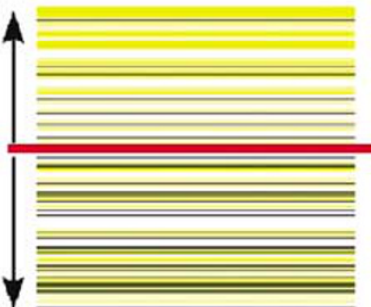

Figure 9 Glial/neuronal enrichment of altered gene expression in three brain areas in the UCMS model. Transcripts in CC $(n=254)$, AMY $(n=299)$, and DG $(n=166)$ are organized vertically according to the extent of altered gene expression. Up and down arrows indicate up- and downregulation of gene transcripts in the UCMS model. Color overlay indicates the cellular origin of the transcript: yellow $\sim$ glial origin $(\mathrm{WM} / \mathrm{GM}>$ I.5), gray $\sim$ neuronal origin $(-1.5<\mathrm{WM} / \mathrm{GM})$, and white $\sim$ both cellular populations $(-1.5<\mathrm{WM} / \mathrm{GM}<1.5)$ (Sibille et al, 2008). Note the apparent increased representation of glial expression in downregulated genes in AMY. Red bar separates up- from downregulated genes.

Table 3 Functional annotation of identified gene sets

\begin{tabular}{|c|c|c|c|c|}
\hline Area & Gene category & Glial/neuronal origin & Functional cluster & \# Groups/cluster \\
\hline & Mood and therapeutic & Neuronal & Cation binding; metal ion binding; ion binding & 3 \\
\hline & & Glial & Intracellular organelle & 4 \\
\hline \multirow[t]{7}{*}{$\mathrm{CC}$} & & & Chromosome organization and biogenesis & 2 \\
\hline & Therapeutic only & Neuronal & Zinc; zinc-finger; zinc ion binding & 3 \\
\hline & & & Regulation of transcription, DNA dependent & 7 \\
\hline & & Glial & Metabolism & 2 \\
\hline & & & Serine/threonine-protein kinase & 8 \\
\hline & Mood and therapeutic & Neuronal & Ion channel activity & $10 P<e-04$ \\
\hline & & & Neuropeptide and neurotransmitter receptor activity & 7 \\
\hline & & Glial & Transmembrane & । \\
\hline & Mood and therapeutic & Neuronal & None & \\
\hline \multirow[t]{5}{*}{ DG } & & Glial & None & \\
\hline & Therapeutic only & Neuronal & None & \\
\hline & & Glial & Regulation of transcription, DNA dependent & 12 \\
\hline & & & Protein amino-acid phosphorylation & 8 \\
\hline & & & Cellular metabolism & 3 \\
\hline
\end{tabular}

To reduce the redundancy of gene ontology (GO) annotations, clusters of $\mathrm{GO}$ groups with $P$-values less than 0.05 and with similar annotations were regrouped (ie, \# groups/clusters; see Materials and methods). Results in DG were either borderline or not significant due to the small number of genes. The only two functional clusters that displayed very low P-values were identified in AMY.

anhedonia-like behaviors), (2) good construct validity (unpredictable 'psycho-social' stress mimics real-life stress etiology and recruits equivalent neuroendocrine systems, (3) good predictive validity (pharmacological reversal by antidepressant treatments), and (4) respect of time courses for mechanisms of disease and drug reversal. On the other hand, alternate paradigms frequently used to either induce or characterize depressive-like states (social defeat, learned 
helpnessness), or to predict of antidepressant activities (forced swim and tail suspension tests), follow only limited numbers of these criteria in modeling depression (Cryan and Holmes, 2005).

\section{Region-Specific and State-Dependent Corticolimbic Transcriptome Changes in a Validated Rodent Model of Depression and of Antidepressant Reversal}

Results from our large-scale gene expression studies suggest that molecular changes following UCMS and/or antidepressant exposure reflect changes in the activity of the respective brain areas, rather than the direct recruitment (by UCMS and/or antidepressant) of common molecular or cellular mechanisms. In other words, although the brain areas investigated participate in a corticolimbic network of mood regulation (Seminowicz et al, 2004; Pezawas et al, 2005), the observed differences in transcriptome changes may reflect brain area differences in terms of intrinsic cellular networks, activation states, information processing, and adaptive mechanisms. Brain area-specific effects of BDNF have been reported in the social defeat stress paradigm (Berton et al, 2006), and area-specific regulation of ERK MAP kinase in UCMS-treated rats (Gourley et al, 2007), but to our knowledge, this has not been investigated on a large-scale. Conti et al (2007) have reported that electro-convulsive therapy, sleep deprivation, and fluoxetine induced transcriptome profiles that mostly differed across brain areas and treatment modalities, although the interpretation of these findings were limited by a lack of statistical validation and by the use of normal control rats.

Accordingly, the present results confirm our hypothesis that antidepressant effects in control animals do not extrapolate to 'depressive states.' Indeed, considering that antidepressants are devoid of mood-changing effects in non-depressed healthy humans, and in view of the poor correlation of transcriptome effects of antidepressant treatments between control and UCMS states (Figure 7), the critical antidepressant-induced modifications in gene regulation appear to be conditional on the presence of a depression-related neuropathology. Here, correlating behavior and physical changes with response to antidepressant treatments and with changes in gene expression in a validated rodent model of depression, we report sets of genes with potential roles in mood regulation and/or therapeutic treatment, and confirm the AMY as a key brain area for investigating the molecular pathology of depression.

\section{Fluoxetine and $\mathrm{CRF}_{1}$ Antagonist Treatments of UCMS-Exposed Mice Induced Similar Physical, Behavioral, and Genomic Profiles}

The uniform reversal of the physical, behavioral, and transcriptome effects of UCMS by two different antidepressants is consistent with clinical data, as antidepressants typically treat the depressive syndrome as a whole, but is surprising from a mechanistic point of view. Indeed, the striking similarities in transcriptome profiles after the two treatments (Figures 6 and 7) strongly suggest that SSR 125543 recapitulates in a brain region-specific manner the overall effects of increased serotonin signaling, and that similar cellular circuitries are ultimately targeted by increased serotonin availability and $\mathrm{CRF}_{1}$ blockade, at least in the areas investigated. The serotonin and CRF systems interact on midbrain serotonergic neurons (Price et al, 1998; Jankowski and Sesack, 2004; Waselus et al, 2005) and $\mathrm{CRF}_{1}$ antagonism could mimic the effect of fluoxetine on serotonin levels (Kirby et al, 2000; Lukkes et al, 2007). Alternatively, fluoxetine and SSR 125543 could regulate a common hormonal system, such as glucocorticoids and the hypothalamic-pituitary-adrenal (HPA) axis, to produce a similar global effect on the brain. HPA abnormalities are common in depression (Holsboer, 2000; Pariante and Miller, 2001) and normalization of HPA function is required for clinical remission in these subjects (Linkowski et al, 1987; Wodarz et al, 1992). Although $\mathrm{CRF}_{1}$ antagonists are generally thought to act directly in various brain regions (Arzt and Holsboer, 2006), $\mathrm{CRF}_{1}$ receptors are in fact the main glucocorticoid secretagogues. Thus, HPA modulation by antagonism of $\mathrm{CRF}_{1}$ receptor or by fluoxetine could represent a common downstream mechanism for both drugs. The direct impact of $\mathrm{CRF}_{1}$ receptor antagonism on the HPA axis could also explain the shorter delay of onset of SSR 125543 on the fur index, although whether this early $\mathrm{CRF}_{1}$ antagonist effect also correlated with early reversals of UCMS-induced behavioral or molecular changes is not known.

\section{Low Gene Expression Plasticity in UCMS Compared to Antidepressant Treatments}

By testing transcriptome changes under several experimental conditions, our studies revealed critical differences in the scope of the transcriptome effects of UCMS and antidepressant treatments, and further suggested differences in drug-induced gene expression plasticity between UCMS or control states. Indeed, UCMS resulted in fewer transcriptional changes in all three brain areas, compared to antidepressant treatments. A potential explanation is that UCMS recruits limited numbers of endogenous neural networks that are involved in the stress response, while drug treatments target indiscriminately much wider systems. Interestingly, antidepressant treatments affected even larger number of genes in the absence of UCMS (Figure 7). This effect is reported in CC, as array data in antidepressant-exposed control mice was only generated in CC, thus we can only speculate that similar contrasts between effects of antidepressant in control and 'depressed' mice would be present elsewhere. Taken together, our results suggest that UCMS exposure reduced the overall gene expression-related plasticity, with the following effect sizes: UCMS $<$ (antidepressant in UCMS) $<$ (antidepressant in control).

From the experimental point of view, these large differences in genes affected by UCMS and antidepressant treatments, combined with the poor conservations of antidepressant effects across brain areas and 'emotional' states, suggest three notable implications for investigating disease mechanisms: first, brain areas and neural networks appear less plastic under UCMS or 'depressed' state compared to a control non-stressed state; second, the effects of antidepressant treatments are much larger than the correlates of UCMS, encompassing much more than a reversal of UCMS effects, and thus do not represent the opposite of a 'depressive' effect for the vast majority of 
genes; third, results from antidepressant treatment in control animals are unlikely to extrapolate to UCMS or 'depressed' states, thus highlighting the relevance of investigating neurobiological mechanisms in the context of a depression-related neuropathology. Finally, characterizing such large-scale effects and interactions between the environment (ie, UCMS) and drug treatments will be a necessary first step in comparing results across studies, for realistic meta-analysis of large-scale gene array studies.

\section{Enriched UCMS- and Antidepressant-Related Molecular Pathology in AMY}

Results presented here suggest a crucial role for AMY in the molecular correlates of UCMS and antidepressant treatments, as AMY changes (1) predicted similar trends for transcripts in CC and DG (Figure 3), (2) displayed highest and most consistent antidepressant reversals of UCMS effects (Figure 5), and (3) suggested distinct glial and neuronal phenotypes consistent with previous reports in depression (Tables 1-3). Relevant biological systems included the GABA (Gabra2 and Gabrg1), glutamate (Grin2a, Grin2b, Grin2c, Grin3a, Gria4, and Grm3-8), serotonin (Htr4), peptide (Igf1r, Igfbp3, Npy, Npy1r, NPY2r, and $M c h r 1$ ), and other (P2rx4 and Ntrk3) neurotransmitter systems, and the phospholipase C (Plch1, Dgkg, and Plcd1), protein kinase C (Akap5-10 and Prkcd), mitogen-activated protein kinase (MAKbp1, Map2k6, Map4k5, Mapk1, and Mapk10), and cyclic adenosine monophosphate (Adcy2 and Pde7a)-related pathways. AMY glial-related changes suggested an antidepressant-sensitive downregulation of oligodendrocyte structure and function (Mobp, Edg2, Gsn, Cnp1, Gpr37, Pllp, Enpp2, Pmp22, Mpzl1 and Plp1; Tables 2 and 3), consistent with prior reports of decreased oligodendrocyte numbers in AMY in human depression (Hamidi et al, 2004) and decreased oligodendrocyte-related gene expression in the adjacent temporal cortex (Aston et al, 2005). These findings were not present in CC and may differ from schizophrenia-related pathology, where more widespread oligodendrocyte-related findings were reported (Haroutunian et al, 2007). Nevertheless, these results raised the question as to the role and contribution of decreased oligodendrocyte function in neuropsychiatric disorders. Widespread decreases in elderly schizophrenic subjects may disrupt communication between multiple brain areas through altered myelination of fiber tracts (Haroutunian et al, 2007), but the more regionally restricted phenotype observed here suggests the presence of a local biological mechanism affecting oligodendrocyte homeostasis. Whether this mechanism occurs at the level of cellular proliferation, maturation, and/or maintenance remains to be investigated.

Functional and pathway analyses of altered gene expression in CC of UCMS-exposed mice revealed a more complex picture involving numerous cellular functions (receptors, signal-transduction pathways, transcription, metabolism, and structural components) and did not a priori identify specific cellular population as in AMY. In contrast, findings in DG were overall more limited in scope. UCMS-induced changes were only marginally reversed by antidepressants, and overall did not provide supporting evidence for reported changes in neurotropic function in depression and/or antidepressant effects in that brain area. Specifically, we observed no changes in $B d n f$ RNA levels after UCMS or antidepressant treatments. This absence of change was confirmed by independent qPCR measurements (not shown). Taken together, these results are consistent with a recent report showing that, in contrast to immobilization stress, UCMS in rats yielded no change in total $B d n f$ mRNA in DG, despite differentially altering specific Bdnf transcripts (Nair et al, 2007). We cannot exclude that similar changes occurred here, as exonic-dependent transcriptional regulation was not assessed in this study, thus keeping potential biological significant mechanisms undetectable (ie, post-translational, protein level, maturation, etc.). An alternate explanation for the paucity of DG findings may be that the UCMS model represents a more appropriate model for the altered mood regulation component of depression, rather than for the cognitive aspects of the disease, which may correlate more closely with changes in stress- or antidepressant-induced modulation of cellular proliferation and neurogenesis in DG (Saxe et al, 2006). Nevertheless, it is important to note that even if more limited in scope, molecular findings in DG may include relevant changes that could be critical to the altered mood-related phenotype. Collecting samples from brain tissue may also lead to missing signals and to underestimating the dynamic range for some changes, due to signal dilution in heterogeneous tissues, such as DG. An alternative approach would be to investigate altered gene expression based on laser-captureselected cell populations (Ginsberg et al, 2004), although this approach requires a priori knowledge as to which cellular population to target, and has the disadvantage of being less quantitative, due to very small amount of RNA collected and partial mRNA decay during the time required for the procedure.

\section{Summary, Limitations}

Taken together, our strategy in this study was to maximize the discovery process by maintaining statistical thresholds at moderate stringency and by relying on cumulative effects over larger groups of genes, as previously demonstrated by the investigation of a robust biological phenomenon such as aging (Sibille et al, 2007b). This approach was helpful here at reliably identifying global features of UCMS and antidepressant treatments, which were otherwise not discernable by other approaches. Specifically, results provided answers to critical questions relating to research on the neurobiology of neuropsychiatric disorder, such as (1) antidepressant drug treatments in 'non-depressed' animals are probably not relevant to the disease and its treatment, (2) mechanisms of disease and drug treatments will differ across brain regions investigated, and (3) overall gene expression plasticity decreased from control to UCMS states. On the other hand, we also suggest that results at the level of single genes should be viewed with caution. Indeed, in the absence of formal control for false discovery and without independent confirmation in other cohorts and models, the microarray results will include false positives for individual genes. Rates of false discovery are difficult to assess due to the overall inter-dependence of genes in biological systems (Lee et al, 2003; Li et al, 2004) and since the observed effects of 'depression' and treatments are 
typically large in the numbers of genes being affected, but modest in the extent of transcript changes and statistical robustness for individual genes (see current results, and Landgrebe et al, 2002; Rausch et al, 2002; Newton et al, 2003; Drigues et al, 2003; Palotas et al, 2004; Altar et al, 2004; Wong et al, 2004; Ploski et al, 2006; Takahashi et al, 2006). In other words, controlling for false discovery would exclude large numbers of genes of interest, while maintaining medium statistical stringency will necessarily include false-positive results. So, while the overall state-dependent and region-specific effects of depression and antidepressant treatments are robust and rely on large number of genes, results for individual genes need to be independently verified. Accordingly, we make available large-scale data (Table 2 and Supplementary Information) so that other groups can compare their results in other cohorts, models, and species. To facilitate the identification of changes that are most relevant to altered mood regulation, and as potential leads for future cell-specific targeted approaches, we are currently comparing the present results to array findings in the homologous brain areas in human depressed subjects. These results will be described elsewhere. Additional limitations of the present study that will also need to be addressed in future studies include (1) correlations of RNA findings with protein levels, (2) which genes and pathways correspond to causative changes in mood regulation, therapeutic reversal, drug side effects or epiphenomena, and (3) the extent to which these findings extrapolate to other antidepressant treatments.

\section{CONCLUSION}

Using a naturalistic animal model of depression, we demonstrate that the gene expression-related effects of two antidepressant treatments (fluoxetine and $\mathrm{CRF}_{1}$ antagonism) are strongly influenced by the intrinsic biology of different brain areas (ie, brain region-specificity), and vary greatly depending on whether treated animals are in a control or 'depressed' state (ie, state-dependency). Correlations between behavioral states, drug exposure, and altered gene transcripts suggested candidate genes and pathways for region-specific contributions to mood regulation and therapeutic improvement, confirmed several prior depression-related findings, and highlighted the critical role of AMY in investigating the molecular pathophysiology of depression.

\section{ACKNOWLEDGEMENTS}

This study was supported by the National Institute of Mental Health or the National Institutes of Health NIMH KO1MH067721 (ES) and 5P50MH066171 (ES). The content is solely the responsibility of the authors and does not necessarily represent the official views of the National Institute of Mental Health or the National Institutes of Health. We thank Sunghee Oh for statistical support. Guy Griebel is an employee of Sanofi-Aventis.

\section{DISCLOSURE/CONFLICT OF INTEREST}

The authors of this paper have no conflict of interests.

\section{REFERENCES}

Alonso R, Griebel G, Pavone G, Stemmelin J, Le Fur G, Soubrie P (2004). Blockade of CRF(1) or V(1b) receptors reverses stressinduced suppression of neurogenesis in a mouse model of depression. Mol Psychiatry 9: 278-286, 224.

Altar CA, Laeng P, Jurata LW, Brockman JA, Lemire A, Bullard J et al (2004). Electroconvulsive seizures regulate gene expression of distinct neurotrophic signaling pathways. J Neurosci 24: 2667-2677.

Arzt E, Holsboer F (2006). CRF signaling: molecular specificity for drug targeting in the CNS. Trends Pharmacol Sci 27: 531-538.

Aston C, Jiang L, Sokolov BP (2005). Transcriptional profiling reveals evidence for signaling and oligodendroglial abnormalities in the temporal cortex from patients with major depressive disorder. Mol Psychiatry 10: 309-322.

Ayensu WK, Pucilowski O, Mason GA, Overstreet DH, Rezvani AH, Janowsky DS (1995). Effects of chronic mild stress on serum complement activity, saccharin preference, and corticosterone levels in Flinders lines of rats. Physiol Behav 57: 165-169.

Banasr M, Valentine GW, Li XY, Gourley SL, Taylor JR, Duman RS (2007). Chronic unpredictable stress decreases cell proliferation in the cerebral cortex of the adult rat. Biol Psychiatry 62: 496-504.

Belmaker RH, Agam G (2008). Major depressive disorder. N Engl J Med 358: 55-68.

Berton O, McClung CA, DiLeone RJ, Krishnan V, Renthal W, Russo SJ et al (2006). Essential role of BDNF in the mesolimbic dopamine pathway in social defeat stress. Science 311: 864-868.

Bowley MP, Drevets WC, Ongur D, Price JL (2002). Low glial numbers in the amygdala in major depressive disorder. Biol Psychiatry 52: 404-412.

Castren E (2004). Neurotrophic effects of antidepressant drugs. Curr Opin Pharmacol 4: 58-64.

Conti B, Maier R, Barr AM, Morale MC, Lu X, Sanna PP et al (2007). Region-specific transcriptional changes following the three antidepressant treatments electro convulsive therapy, sleep deprivation and fluoxetine. Mol Psychiatry 12: 167-189.

Cryan JF, Holmes A (2005). The ascent of mouse: advances in modelling human depression and anxiety. Nat Rev Drug Discov 4: 775-790.

Dennis Jr G, Sherman BT, Hosack DA, Yang J, Gao W, Lane HC et al (2003). DAVID: Database for Annotation, Visualization, and Integrated Discovery. Genome Biol 4: 3.

Drevets WC, Price JL, Simpson Jr JR, Todd RD, Reich T, Vannier M et al (1997). Subgenual prefrontal cortex abnormalities in mood disorders. Nature 386: 824-827.

Drigues N, Poltyrev T, Bejar C, Weinstock M, Youdim MB (2003). cDNA gene expression profile of rat hippocampus after chronic treatment with antidepressant drugs. J Neural Transm 110: 1413-1436.

Ducottet C, Aubert A, Belzung C (2004). Susceptibility to subchronic unpredictable stress is related to individual reactivity to threat stimuli in mice. Behav Brain Res 155: 291-299.

Ducottet C, Griebel G, Belzung C (2003). Effects of the selective nonpeptide corticotropin-releasing factor receptor 1 antagonist antalarmin in the chronic mild stress model of depression in mice. Prog Neuropsychopharmacol Biol Psychiatry 27: 625-631.

Duman RS, Heninger GR, Nestler EJ (1997). A molecular and cellular theory of depression. Arch Gen Psychiatry 54: 597-606.

Duman RS, Malberg J, Thome J (1999). Neural plasticity to stress and antidepressant treatment. Biol Psychiatry 46: 1181-1191.

Erraji-BenChekroun L, Underwood MD, Arango V, Galfalvy HC, Pavlidis P, Smyrniotopoulos P et al (2005). Molecular aging in human prefrontal cortex is selective and continuous throughout adult life. Biol Psychiatry 57: 549-558. 
Galfalvy HC, Erraji-Benchekroun L, Smyrniotopoulos P, Pavlidis P, Ellis SP, Mann JJ et al (2003). Sex genes for genomic analysis in human brain: internal controls for comparison of probe level data extraction. BMC Bioinformatics 4: 37.

Ginsberg SD, Elarova I, Ruben M, Tan F, Counts SE, Eberwine JH et al (2004). Single-cell gene expression analysis: implications for neurodegenerative and neuropsychiatric disorders. Neurochem Res 29: 1053-1064.

Gourley SL, Wu FJ, Kiraly DD, Ploski JE, Kedves AT, Duman RS et al (2007). Regionally specific regulation of ERK MAP kinase in a model of antidepressant-sensitive chronic depression. Biol Psychiatry 4: 353-359.

Griebel G, Simiand J, Steinberg R, Jung M, Gully D, Roger P et al (2002). 4-(2-Chloro-4-methoxy-5-methylphenyl)- $N$-[(1S)-2-cyclopropyl-1-(3-fluoro-4-methylp henyl)ethyl]5-methyl- $N$-(2-propynyl)1,3-thiazol-2-amine hydrochloride (SSR125543A), a potent and selective corticotrophin-releasing factor(1) receptor antagonist. II. Characterization in rodent models of stress-related disorders. J Pharmacol Exp Ther 301: 333-345.

Guillot PV, Roubertoux PL, Crusio WE (1994). Hippocampal mossy fiber distributions and intermale aggression in seven inbred mouse strains. Brain Res 660: 167-169.

Hamidi M, Drevets WC, Price JL (2004). Glial reduction in amygdala in major depressive disorder is due to oligodendrocytes. Biol Psychiatry 55: 563-569.

Haroutunian V, Katsel P, Dracheva S, Stewart DG, Davis KL (2007). Variations in oligodendrocyte-related gene expression across multiple cortical regions: implications for the pathophysiology of schizophrenia. Int J Neuropsychopharmacol 10: 565-573.

Holsboer F (2000). The corticosteroid receptor hypothesis of depression. Neuropsychopharmacology 23: 477-501.

Irizarry RA, Bolstad BM, Collin F, Cope LM, Hobbs B, Speed TP (2003). Summaries of Affymetrix GeneChip probe level data. Nucleic Acids Res 31: e15.

Ising M, Zimmermann US, Kunzel HE, Uhr M, Foster AC, Learned-Coughlin SM et al (2007). High-affinity CRF1 receptor antagonist NBI-34041: preclinical and clinical data suggest safety and efficacy in attenuating elevated stress response. Neuropsychopharmacology 32: 1941-1949.

Jankowski MP, Sesack SR (2004). Prefrontal cortical projections to the rat dorsal raphe nucleus: ultrastructural features and associations with serotonin and gamma-aminobutyric acid neurons. J Comp Neurol 468: 518-529.

Kirby LG, Rice KC, Valentino RJ (2000). Effects of corticotropinreleasing factor on neuronal activity in the serotonergic dorsal raphe nucleus. Neuropsychopharmacology 22: 148-162.

Kulkarni SK, Dhir A (2007). Effect of various classes of antidepressants in behavioral paradigms of despair. Prog Neuropsychopharmacol Biol Psychiatry 31: 1248-1254.

Kunzel HE, Zobel AW, Nickel T, Ackl N, Uhr M, Sonntag A et al (2003). Treatment of depression with the CRH-1-receptor antagonist R121919: endocrine changes and side effects. J Psychiatr Res 37: 525-533.

Landgrebe J, Welzl G, Metz T, van Gaalen MM, Ropers H, Wurst W et al (2002). Molecular characterisation of antidepressant effects in the mouse brain using gene expression profiling. J Psychiatr Res 36: 119-129.

Lee HK, Hsu AK, Sajdak J, Qin J, Pavlidis P (2003). Coexpression analysis of human genes across many microarray data sets. Genome Res 14: 1085-1094.

Li KC, Liu CT, Sun W, Yuan S, Yu T (2004). A system for enhancing genome-wide coexpression dynamics study. Proc Natl Acad Sci USA 101: 15561-15566.

Li S, Wang C, Wang M, Li W, Matsumoto K, Tang Y (2007). Antidepressant like effects of piperine in chronic mild stress treated mice and its possible mechanisms. Life Sci 80: 1373-1381.

Linkowski P, Mendlewicz J, Kerkhofs M, Leclercq R, Golstein J, Brasseur M et al (1987). 24-Hour profiles of adrenocortico- tropin, cortisol, and growth hormone in major depressive illness: effect of antidepressant treatment. J Clin Endocrinol Metab 65: 141-152.

Lukkes JL, Forster GL, Renner KJ, Summers CH (2007). Corticotropin-releasing factor 1 and 2 receptors in the dorsal raphe differentially affect serotonin release in the nucleus accumbens. Eur J Pharmacol 578: 185-193.

Manji HK, Drevets WC, Charney DS (2001). The cellular neurobiology of depression. Nat Med 7: 541-547.

Mayberg HS (1997). Limbic-cortical dysregulation: a proposed model of depression. J Neuropsychiatry Clin Neurosci 9: 471-481.

Mineur YS, Belzung C, Crusio WE (2006). Effects of unpredictable chronic mild stress on anxiety and depression-like behavior in mice. Behav Brain Res 175: 43-50.

Mineur YS, Prasol DJ, Belzung C, Crusio WE (2003). Agonistic behavior and unpredictable chronic mild stress in mice. Behav Genet 33: 513-519.

Nair A, Vadodaria KC, Banerjee SB, Benekareddy M, Dias BG, Duman RS et al (2007). Stressor-specific regulation of distinct brain-derived neurotrophic factor transcripts and cyclic AMP response element-binding protein expression in the postnatal and adult rat hippocampus. Neuropsychopharmacology 32: 1504-1519.

Nestler EJ, Barrot M, DiLeone RJ, Eisch AJ, Gold SJ, Monteggia LM (2002). Neurobiology of depression. Neuron 34: 13-25.

Newton SS, Collier EF, Hunsberger J, Adams D, Terwilliger R, Selvanayagam E et al (2003). Gene profile of electroconvulsive seizures: induction of neurotrophic and angiogenic factors. J Neurosci 23: 10841-10851.

Palotas M, Palotas A, Puskas LG, Kitajka K, Pakaski M, Janka Z et al (2004). Gene expression profile analysis of the rat cortex following treatment with imipramine and citalopram. Int $J$ Neuropsychopharmacol 7: 401-413.

Pariante CM, Miller AH (2001). Glucocorticoid receptors in major depression: relevance to pathophysiology and treatment. Biol Psychiatry 49: 391-404.

Paxinos G, Franklin KBJ (2001). The Mouse Brain in Stereotoxic Coordinates. Academic Press: San Diego, CA.

Pezawas L, Meyer-Lindenberg A, Drabant EM, Verchinski BA, Munoz KE, Kolachana BS et al (2005). 5-HTTLPR polymorphism impacts human cingulate-amygdala interactions: a genetic susceptibility mechanism for depression. Nat Neurosci 8: 828-834.

Ploski JE, Newton SS, Duman RS (2006). Electroconvulsive seizureinduced gene expression profile of the hippocampus dentate gyrus granule cell layer. J Neurochem 99: 1122-1132.

Pothion S, Bizot JC, Trovero F, Belzung C (2004). Strain differences in sucrose preference and in the consequences of unpredictable chronic mild stress. Behav Brain Res 155: 135-146.

Price ML, Curtis AL, Kirby LG, Valentino RJ, Lucki I (1998). Effects of corticotropin-releasing factor on brain serotonergic activity. Neuropsychopharmacology 18: 492-502.

Rajkowska G, Miguel-Hidalgo JJ, Wei J, Dilley G, Pittman SD, Meltzer HY et al (1999). Morphometric evidence for neuronal and glial prefrontal cell pathology in major depression. Biol Psychiatry 45: 1085-1098.

Rausch JL, Gillespie CF, Fei Y, Hobby HM, Stoming T, Ganapathy $\mathrm{V}$ et al (2002). Antidepressant effects on kinase gene expression patterns in rat brain. Neurosci Lett 334: 91-94.

Santarelli L, Saxe M, Gross C, Surget A, Battaglia F, Dulawa S et al (2003). Requirement of hippocampal neurogenesis for the behavioral effects of antidepressants. Science 301: 805-809.

Saxe MD, Battaglia F, Wang JW, Malleret G, David DJ, Monckton JE et al (2006). Ablation of hippocampal neurogenesis impairs contextual fear conditioning and synaptic plasticity in the dentate gyrus. Proc Natl Acad Sci USA 103: 17501-17506.

Seminowicz DA, Mayberg HS, McIntosh AR, Goldapple K, Kennedy S, Segal Z et al (2004). Limbic-frontal circuitry in 
major depression: a path modeling metaanalysis. Neuroimage 22: 409-418.

Sheline YI, Gado MH, Price JL (1998). Amygdala core nuclei volumes are decreased in recurrent major depression. NeuroReport 9: 2023-2028.

Shelton RC (2007). The molecular neurobiology of depression. Psychiatr Clin North Am 30: 1-11.

Sibille E, Arango V, Joeyen-Waldorf J, Wang Y, Leman S, Surget A et al (2008). Large-scale estimates of cellular origins of nRNAs: enhancing the yield of transcriptome analyses. Neurobiol Dis 167: 198-206.

Sibille E, Su J, Leman S, Le Guisquet AM, Ibarguen-Vargas Y, JoeyenWaldorf J et al (2007). Lack of serotonin(1B) receptor expression leads to age-related motor dysfunction, early onset of brain molecular aging and reduced longevity. Mol Psychiatry 12: 1042-1056.

Surget A, Saxe M, Leman S, Ibarguen-Vargas Y, Chalon S, Griebel $\mathrm{G}$ et al (2008). Drug-dependent requirement of hippocampal neurogenesis in a model of depression and of antidepressant reversal. Biol Psychiatry 2008 Apr 10 [E-pub ahead of print] doi: 10.1016/j.biopsych.2008.02.022.

Takahashi Y, Washiyama K, Kobayashi T, Hayashi S (2006). Gene expression in the brain from fluoxetine-injected mouse using DNA microarray. Ann NY Acad Sci 1074: 42-51.

Tsankova NM, Berton O, Renthal W, Kumar A, Neve RL, Nestler EJ (2006). Sustained hippocampal chromatin regulation in a mouse model of depression and antidepressant action. Nat Neurosci 9: 519-525.
Waselus M, Valentino RJ, Van Bockstaele EJ (2005). Ultrastructural evidence for a role of gamma-aminobutyric acid in mediating the effects of corticotropin-releasing factor on the rat dorsal raphe serotonin system. J Comp Neurol 482: 155-165.

Willner P (2005). Chronic mild stress (CMS) revisited: consistency and behavioural-neurobiological concordance in the effects of CMS. Neuropsychobiology 52: 90-110.

Wodarz N, Rupprecht R, Kornhuber J, Schmitz B, Wild K, Riederer P (1992). Cell-mediated immunity and its glucocorticoid-sensitivity after clinical recovery from severe major depressive disorder. J Affect Disord 25: 31-38.

Wong ML, Licinio J (2001). Research and treatment approaches to depression. Nat Rev Neurosci 2: 343-351.

Wong ML, O'Kirwan F, Hannestad JP, Irizarry KJ, Elashoff D, Licinio J (2004). St John's wort and imipramine-induced gene expression profiles identify cellular functions relevant to antidepressant action and novel pharmacogenetic candidates for the phenotype of antidepressant treatment response. Mol Psychiatry 9: 237-251.

Yalcin I, Aksu F, Belzung C (2005). Effects of desipramine and tramadol in a chronic mild stress model in mice are altered by yohimbine but not by pindolol. Eur J Pharmacol 514: 165-174.

Zobel AW, Nickel T, Kunzel HE, Ackl N, Sonntag A, Ising M et al (2000). Effects of the high-affinity corticotropin-releasing hormone receptor 1 antagonist R121919 in major depression: the first 20 patients treated. $J$ Psychiatr Res 34: 171-181.

Supplementary Information accompanies the paper on the Neuropsychopharmacology website (http://www.nature.com/npp) 